\title{
A VARIATIONAL PROBLEM FOR COUPLES OF FUNCTIONS AND MULTIFUNCTIONS WITH INTERACTION BETWEEN LEAVES
}

\author{
Emilio Acerbi ${ }^{1}$, Gianluca Crippa ${ }^{1}$ And Domenico MucCi $^{1}$
}

\begin{abstract}
We discuss a variational problem defined on couples of functions that are constrained to take values into the 2-dimensional unit sphere. The energy functional contains, besides standard Dirichlet energies, a non-local interaction term that depends on the distance between the gradients of the two functions. Different gradients are preferred or penalized in this model, in dependence of the sign of the interaction term. In this paper we study the lower semicontinuity and the coercivity of the energy and we find an explicit representation formula for the relaxed energy. Moreover, we discuss the behavior of the energy in the case when we consider multifunctions with two leaves rather than couples of functions.
\end{abstract}

Mathematics Subject Classification. 49Q20, 54C60.

Received May 2, 2011.

Published online 16 January 2012.

\section{INTRODUCTION}

\section{The Dirichlet energy}

In the last decades there has been a growing interest in variational problems for vector valued mappings with geometric constraints, as e.g. for mappings defined between smooth manifolds isometrically embedded in Euclidean spaces. The most studied one is perhaps the minimization problem of the Dirichlet energy

$$
\mathbf{D}(u):=\frac{1}{2} \int_{B^{n}}|D u(x)|^{2} \mathrm{~d} x
$$

for maps $u: B^{n} \rightarrow \mathbb{R}^{3}$, where $B^{n}$ is the unit ball in $\mathbb{R}^{n}$, that are constrained to take values into the unit sphere $\mathbb{S}^{2}$ of $\mathbb{R}^{3}$. The problem is naturally set in the Sobolev class

$$
W^{1,2}\left(B^{n}, \mathbb{S}^{2}\right):=\left\{u \in W^{1,2}\left(B^{n}, \mathbb{R}^{3}\right):|u(x)|=1 \text { for a.e. } x \in B^{n}\right\} .
$$

In the physical model $n=3$, the above problem is related to the theory of liquid crystals [7], Vol. II, Section 5.1. Namely, the function $u$ represents the direction of the symmetry axis of the rod-like molecules of the liquid crystal, and the minimization of the Dirichlet integral $\mathbf{D}(u)$ forces the molecules to organize

\footnotetext{
Keywords and phrases. Relaxed energies, multifunctions, Cartesian currents.

1 Dipartimento di Matematica dell'Università di Parma, Parco Area delle Scienze 53/A, 43124 Parma, Italy.

emilio.acerbi@unipr.it; gianluca.crippa@unipr.it; domenico.mucci@unipr.it
} 
themselves in such a way that their symmetry axes are as aligned as possible, compatibly with the assigned boundary conditions.

It is well known that during the process of relaxation in $W^{1,2}\left(B^{n}, \mathbb{S}^{2}\right)$ the functional $\mathbf{D}(u)$ may produce energy concentration. In dimension $n=2$, this corresponds to the so called bubbling-off of spheres: the energy concentrates in a quantized way around points, compare [10]. As a consequence, geometric invariants such as the degree of mappings from $B^{2}$ into $\mathbb{S}^{2}$ may not be preserved by the sequential weak $W^{1,2}$-convergence.

Moreover, in the physical model $n=3$, the weak $W^{1,2}$-limits of sequences of smooth maps $u_{k}$ from $B^{3}$ into $\mathbb{S}^{2}$ with equibounded Dirichlet energies, $\sup _{k} \mathbf{D}\left(u_{k}\right)<\infty$, may in general be Sobolev maps $u \in W^{1,2}\left(B^{3}, \mathbb{S}^{2}\right)$ with point singularities that are positioned in correspondence with "holes" in the graph of $u$, as e.g. for the map $u(x):=x /|x|$, see Example 3.1. On the other hand, Brezis-Coron-Lieb showed in the relevant paper [3] that energy minimizing maps $\bar{u}$ in $W^{1,2}\left(B^{3}, \mathbb{S}^{2}\right)$ have isolated singular points $a_{i}$ of degree \pm 1 , and near such points $a_{i}$ we have

$$
\bar{u}(x) \simeq \pm R \frac{x-a}{|x-a|},
$$

where $R$ is a rotation in $\mathbb{S}^{2}$.

\section{Cartesian currents}

The theory of Cartesian currents, introduced by Giaquinta-Modica-Souček in 1989, and extensively studied in the monograph [7], has revealed to be a satisfactory approach to deal with geometric problems such as the liquid crystal energy, especially in higher dimension $n$.

The naive idea is to look at a map $u$ in $W^{1,2}\left(B^{n}, \mathbb{S}^{2}\right)$ as a current $G_{u}$ carried by its graph. The Dirichlet energy is extended to the class of integer multiplicity rectifiable currents in $\mathcal{R}_{n}\left(B^{n} \times \mathbb{S}^{2}\right)$ that naturally arise as weak limits of sequences of graphs $G_{u_{k}}$ of smooth maps $u_{k}: B^{n} \rightarrow \mathbb{S}^{2}$ with equibounded Dirichlet energies. The weak limit currents describe the energy concentration phenomenon, and preserve geometric invariants as the degree.

A natural setting is therefore the class cart ${ }^{2,1}\left(B^{n} \times \mathbb{S}^{2}\right)$ of Cartesian currents, see Definition 3.2 below. Roughly speaking, a current $T$ in $\operatorname{cart}^{2,1}\left(B^{n} \times \mathbb{S}^{2}\right)$ is given by the sum of a graph $G_{u_{T}}$, for some $u_{T} \in W^{1,2}\left(B^{n}, \mathbb{S}^{2}\right)$, and a vertical current of the type $L_{T} \times \llbracket \mathbb{S}^{2} \rrbracket$, where $L_{T}$ is an integer multiplicity rectifiable current of codimension 2 in $B^{n}$ (that is, a sum of signed unit Dirac masses in the 2-dimensional case, and of oriented lines in the physically relevant 3-dimensional case). Again very roughly speaking, this $L_{T}$ closes the holes created by the relevant singularities, i.e. those with nonzero degree. Being the weak limit of smooth graphs, the current $T$ has no boundary inside $B^{n} \times \mathbb{S}^{2}$. This yields that in higher dimension $n \geq 3$ the current $L_{T}$ "encloses" the codimension 3 singularities of the map $u_{T}$ : if $n=3$ and $u_{T}(x)=x /|x|$, then $L_{T}$ may be given by (the current integration of 1-forms in $B^{3}$ over) an oriented rectifiable arc with initial point at the boundary $\partial B^{3}$ and final point at the origin of $B^{3}$, see Example 3.5.

The Dirichlet energy functional $T \mapsto \mathbf{D}(T)$ is well defined and lower semicontinuous in the class $\operatorname{cart}^{2,1}\left(B^{n} \times\right.$ $\mathbb{S}^{2}$ ), and it extends the Dirichlet integral of mappings in the sense that $\mathbf{D}(T)=\mathbf{D}(u)$ if $T=G_{u}$. Moreover, a strong density property of smooth maps holds. This approach allows to describe in a satisfactory way the relaxed Dirichlet energy of maps in $W^{1,2}\left(B^{n}, \mathbb{S}^{2}\right)$.

All the above generalizes to the case when $B^{n}$ is replaced by a sufficiently regular open set $\Omega \subset \mathbb{R}^{n}$. For a quick review of these results the reader is addressed to Section 3; for more details we refer to [7,9], and for various physical aspects of the theory of liquid crystal we suggest [14].

\section{Energy of couples of functions}

In this paper we are interested in a related variational problem. Instead of considering just one function, we assume that the behavior of our material is characterized by two relevant axes, thus we shall deal with couples $(u, v)$ of $W^{1,2}$-maps defined on the unit ball $B^{n}$ of $\mathbb{R}^{n}$ (with $n \geq 2$ ), and with values in $\mathbb{S}^{2}$, the unit 2-sphere in $\mathbb{R}^{3}$. This could model for instance the behavior of a mixture of two liquid crystals put in the same region. 
The energy we want to study consists of two terms. The first one takes into account the Dirichlet energies of the functions $u$ and $v$, and can physically be seen as the non-interacting part: some energy is required in order to deviate from a parallel alignment for each of the two axes, separately. The second term encodes the interaction between the directions of the two axes: we assume that interactions between the directions happen only up to a certain distance $r$ (which, as we are in the case of the unit ball, will always be understood to be a positive number not exceeding 2 , the diameter of the ball) and depend on the difference between the gradients of the directions. Thus we are led to consider energies of the form

$$
\begin{aligned}
\mathcal{E}(u, v):= & \frac{1}{2} \int_{B^{n}}|D u(x)|^{2} \mathrm{~d} x+\frac{1}{2} \int_{B^{n}}|D v(x)|^{2} \mathrm{~d} x \\
& +\int_{B^{n}} f_{\widetilde{B}_{r}(x)} \frac{1}{2}[f(D u(x)-D v(y))+f(D v(x)-D u(y))] \mathrm{d} y \mathrm{~d} x
\end{aligned}
$$

with $u, v: B^{n} \rightarrow \mathbb{S}^{2}$. Here and in the sequel, $f_{A}:=\frac{1}{|A|} \int_{A}$ denotes the averaged integral and

$$
\widetilde{B}_{r}(x):=\bar{B}_{r}(x) \cap B^{n}, \quad x \in B^{n}, \quad r>0,
$$

where $\bar{B}_{r}(x)$ is the closed ball in $\mathbb{R}^{n}$ of radius $r$ centered at $x$.

In this paper we confine ourselves to the model case where $f(A)=\gamma|A|^{2}$ for some $\gamma \in \mathbb{R}$. The energy we will investigate is thus

$$
\begin{aligned}
\mathcal{E}_{\gamma}(u, v):= & \frac{1}{2} \int_{B^{n}}|D u(x)|^{2} \mathrm{~d} x+\frac{1}{2} \int_{B^{n}}|D v(x)|^{2} \mathrm{~d} x \\
& +\gamma \int_{B^{n}} f_{\widetilde{B}_{r}(x)} \frac{1}{2}\left(|D u(x)-D v(y)|^{2}+|D v(x)-D u(y)|^{2}\right) \mathrm{d} y \mathrm{~d} x .
\end{aligned}
$$

Notice that, depending on the sign of the coefficient $\gamma$ in the interaction term, different gradients of the axes directions may be either preferred or disfavored in this model.

In the sequel we shall discuss the following relaxation of the energy (1.4) on couples of functions in $W^{1,2}\left(B^{n}, \mathbb{S}^{2}\right)$ :

$$
\widetilde{\mathcal{E}_{\gamma}}(u, v):=\inf \left\{\liminf _{k \rightarrow \infty} \mathcal{E}_{\gamma}\left(u_{k}, v_{k}\right) \quad \mid \quad\left\{u_{k}\right\},\left\{v_{k}\right\} \subset C^{\infty}\left(B^{n}, \mathbb{S}^{2}\right), \quad u_{k} \rightarrow u, v_{k} \rightarrow v \text { weakly in } W^{1,2}\right\} .
$$

We observe that one might assume that the interaction depends also on the difference between the directions of the axes, i.e., it contains terms as

$$
\gamma^{\prime} \int_{B^{n}}|u(x)-v(x)|^{2} \mathrm{~d} x \quad \text { or } \quad \gamma^{\prime} \int_{B^{n}} f_{\widetilde{B}_{r}(x)} \frac{1}{2}\left(|u(x)-v(y)|^{2}+|v(x)-u(y)|^{2}\right) \mathrm{d} y \mathrm{~d} x .
$$

However, this kind of interaction term is continuous with respect to the weak $W^{1,2}$-convergence in both entries, so it is preserved in the process of relaxation, without giving rise to any new term.

\section{Energy of multifunctions}

A further natural question regards the possibility of interchanging the two axes $u$ and $v$ starting from which we compute the interaction energy. Assume that at every point $x \in B^{n}$ we only know the set $\{u(x), v(x)\}$, but not which of the unit vectors in such set "comes from $u$ " and which one "comes from $v$ ". We would like to consider an energy whose form is based on (1.4), but depending only on the set $\{u(x), v(x)\}$.

A suitable setting is that of multifunctions with two values. We identify different couples $(u, v)$ and $(\widetilde{u}, \widetilde{v})$ of Sobolev functions in $W^{1,2}\left(B^{3}, \mathbb{S}^{2}\right)$ provided that the set of points $x$ in the domain $B^{3}$ such that the sets $\{u(x), v(x)\}$ and $\{\widetilde{u}(x), \widetilde{v}(x)\}$ are different has measure zero. 
Due to the presence of the non-local interaction term, it turns out that the energy $\mathcal{E}_{\gamma}(u, v)$ may depend on the chosen representative of the equivalence class. An explicit example of such phenomenon is constructed in Section 9. We thus define the energy $\mathcal{E}_{\gamma}[u, v]$ of the multifunction $[u, v]$ through an additional minimization procedure, that is

$$
E_{\gamma}[u, v]:=\inf \left\{\mathcal{E}_{\gamma}(\widetilde{u}, \widetilde{v}) \mid(\widetilde{u}, \widetilde{v}) \sim(u, v)\right\} .
$$

\section{Further related models}

An interesting related energy would be the one with pointwise interaction, instead of short-range interaction. Passing formally to the limit as $r \rightarrow 0$ in (1.4), the pointwise interaction turns out to be expressed by the energy functional

$$
\mathcal{E}_{\gamma}^{0}(u, v)=\frac{1}{2} \int_{B^{n}}|D u(x)|^{2} \mathrm{~d} x+\frac{1}{2} \int_{B^{n}}|D v(x)|^{2} \mathrm{~d} x+\gamma \int_{B^{n}}|D u(x)-D v(x)|^{2} \mathrm{~d} x .
$$

Although some of our results could be obtained also for this energy, the main trouble in dealing with the functional $\mathcal{E}_{\gamma}^{0}$ is due to the strong difficulties in showing its lower semicontinuity (remember that $\gamma$ may be negative). The coercivity of $\mathcal{E}_{\gamma}^{0}$ will be briefly addressed in Remark 5.4.

We remark that another physically interesting analysis, which we do not carry out in this paper, would involve the study of a functional with rigid interaction, e.g. when considering the energy

$$
\frac{1}{2} \int_{B^{n}}|D u(x)|^{2} \mathrm{~d} x+\frac{1}{2} \int_{B^{n}}|D v(x)|^{2} \mathrm{~d} x
$$

under a constraint on the angle between the two physical axes, for instance an orthogonality relation

$$
u(x) \cdot v(x)=0 \quad \text { for a.e. } \quad x \in B^{n} .
$$

\section{Extensions to maps with values into manifolds}

Our results extend in a straightforward way to the class of Sobolev functions $W^{1,2}\left(B^{n}, \mathcal{Y}\right)$, where $\mathcal{Y}$ is any smooth oriented compact manifold isometrically embedded in some Euclidean space. In fact, since the Dirichlet energy $\mathbf{D}(T)$ is lower semicontinuous in the class of Cartesian currents cart ${ }^{2,1}\left(B^{n} \times \mathcal{Y}\right)$, we only have to require that the target manifold satisfies the hypothesis of the density theorem in [8], see also [9], Chapter 5. Of course, the relaxed energy of the functional (1.4) would have a more complicate structure, as for the relaxed Dirichlet energy of maps in $W^{1,2}\left(B^{n}, \mathcal{Y}\right)$, see [9], Section 5.6, but its representation formula is obtained arguing exactly as in the model case $\mathcal{Y}=\mathbb{S}^{2}$. For this reason we omit any further detail, and we only remark that the new lower semicontinuity and coercivity thresholds $\gamma_{s}(r)$ and $\gamma_{c}(r)$ are the same as those we have obtained, for any choice of the target manifold $\mathcal{Y}$ as above, and only depend on the radius $r$ and possibly on the dimension $n$ of the domain. We may also consider a general domain $\Omega$ in place of $B^{n}$, and in such general case the results will also depend on the curvature and shape parameters of the domain $\Omega$ itself.

\section{Plan of the paper}

In Section 2 we present some explicit computations in the 3-dimensional case, underlining the effect of the relaxation of the energy. In Section 3 we review some classical facts from the theory of Cartesian currents, focusing in particular on the theory related to the Dirichlet integral and its relaxation to the class of Cartesian currents cart ${ }^{2,1}\left(B^{n} \times \mathbb{S}^{2}\right)$. Starting with Section 4 we begin our analysis of the energy $\mathcal{E}_{\gamma}(u, v)$. We introduce the natural extension $\mathcal{E}_{\gamma}\left(T_{1}, T_{2}\right)$ of the functional to couples of currents in cart ${ }^{2,1}\left(B^{n} \times \mathbb{S}^{2}\right)$ and study its lower semicontinuity properties. We obtain the existence of a semicontinuity threshold $\gamma_{s}(r)<0$ such that the functional is lower semicontinuous (along couples of sequences of currents with equibounded Dirichlet energies) for every $\gamma \geq \gamma_{s}(r)$. In Section 5 we study the coercivity of the energy, obtaining the existence of a coercivity threshold $\gamma_{c}(r)<0$ such that for every $\gamma>\gamma_{c}(r)$ there exists a constant $C=C(\gamma, r)>0$ such that $\mathcal{E}_{\gamma}(u, v) \geq C(\mathbf{D}(u)+\mathbf{D}(v))$. In Section 6 we finally show a representation formula for the relaxed energy together 
with some approximation and density results. Section 7 is devoted to some comments on the 2-dimensional case and to some examples showing the lack of sharpness of the lower semicontinuity and coercivity thresholds. Section 8 introduces the notion of multifunction and the related expression for the energy; we also show a relaxation formula for such energy. The final Section 9 shows through an example the dependence of the energy on the particular representative in the equivalence class defining the multifunction, thus motivating the definition of the energy of multifunctions given in the previous section.

\section{INTRODUCTORY EXAMPLES}

In this first section we collect some examples and we perform some explicit computations in particular cases, in order to introduce the reader to the topics presented in the rest of the paper. We illustrate in particular the effect of the relaxation of the energy functional, both in the case of couples of functions and in the case of multifunctions. We construct these examples in the (physically relevant) 3-dimensional setting.

\section{The relaxed energy}

We first comment on the effect of the relaxation procedure on the expression of the energy $\mathcal{E}_{\gamma}(u, v)$ (recall the definition of the relaxed energy $\widetilde{\mathcal{E}_{\gamma}}(u, v)$ given in (1.5)). Later in this paper we shall detect the lower semicontinuity and coercivity thresholds $\gamma_{s}(r)$ and $\gamma_{c}(r)$, and for $\gamma$ grater than both thresholds we will prove in Theorem 6.1 that

$$
\widetilde{\mathcal{E}_{\gamma}}(u, v)=\mathcal{E}_{\gamma}(u, v)+\mathcal{G}_{\gamma}(u, v) .
$$

The relaxation process originates a non-negative gap $\mathcal{G}_{\gamma}(u, v)$, explicitly given by the minimization formula

$$
\mathcal{G}_{\gamma}(u, v):=\inf _{L_{u}, L_{v}}\left\{\Upsilon_{\gamma}\left(L_{u}\right)+\Upsilon_{\gamma}\left(L_{v}\right)\right\}
$$

In (2.1), the infimum is computed among all possible curves $L_{u}$ and $L_{v}$ which separately close the singularities of $u$ and $v$, or more precisely such that both $G_{u}+L_{u} \times \llbracket \mathbb{S}^{2} \rrbracket$ and $G_{v}+L_{v} \times \llbracket \mathbb{S}^{2} \rrbracket$ are Cartesian currents.

The functional $\Upsilon_{\gamma}(L)$ is given by $(1+\gamma)$ times the length (with multiplicity) of $L$ plus an extra term which depends on the local interaction. If $L$ is a curve with multiplicity one, we have

$$
\Upsilon_{\gamma}(L)=(1+\gamma) \mathcal{H}^{1}(L)+\gamma \int_{B^{3}} \frac{\mathcal{H}^{1}\left(L \cap \bar{B}_{r}(x)\right)}{\left|\widetilde{B}_{r}(x)\right|} \mathrm{d} x
$$

where, we recall, $\widetilde{B}_{r}(x)$ is given by (1.3). As an example, if $L$ is a segment whose distance from the boundary of $B^{3}$ is greater than $2 r$, then

$$
\Upsilon_{\gamma}(L)=(1+2 \gamma) \mathcal{H}^{1}(L)
$$

whereas in general (see the computation in $(6.3)$ ) we have

$$
\Upsilon_{\gamma}(L)=\int_{L}\left(1+\gamma+\gamma k_{r}(x)\right) \mathrm{d} \mathcal{H}^{1}(x)
$$

where the weight $0<k_{r} \leq 2$, which will be defined in (5.1), takes value 1 if $\|x\| \leq 1-2 r$. We remark that $\max \left\{\gamma_{s}(r), \gamma_{c}(r)\right\}>-1 / 3$, so in any case $\Upsilon_{\gamma}$ will be positive.

In the following two examples we compute explicitly the value of the gap $\mathcal{G}_{\gamma}(u, v)$ in some special cases.

Example 2.1. Let $0 \leq a<1$ and let $u_{a}^{+}$and $u_{a}^{-}$be the functions from $B^{3}$ into $\mathbb{S}^{2}$ defined by

$$
u_{a}^{+}(x):=\frac{\left(x_{1}, x_{2}, x_{3}-a\right)}{\left|\left(x_{1}, x_{2}, x_{3}-a\right)\right|}, \quad u_{a}^{-}(x):=\frac{\left(x_{1}, x_{2},-x_{3}-a\right)}{\left|\left(x_{1}, x_{2},-x_{3}-a\right)\right|} .
$$


Each $u_{a}^{ \pm}$belongs to $W^{1,2}\left(B^{3}, \mathbb{S}^{2}\right)$, and is smooth outside the point $P_{a}^{ \pm}:=(0,0, \pm a)$, where it has one point singularity of degree \pm 1 : indeed, $u_{a}^{+}$is the projection from $(0,0, a)$ whereas $u_{a}^{-}$is the $x_{3}$-symmetric of the projection from $(0,0,-a)$.

Assume that both $a$ and $r$ are very small. In the case $a=0$, the gap (2.1) is estimated by

$$
\mathcal{G}_{\gamma}\left(u_{0}^{+}, u_{0}^{-}\right) \geq 2(1+2 \gamma)(1-2 r)
$$

because each of $L_{u_{0}^{ \pm}}$has to connect the origin to the boundary of the ball. Analogously, in the case $a>0$ small, namely $2 a<1-2 r$, we have

$$
\mathcal{G}_{\gamma}\left(u_{a}^{+}, u_{a}^{+}\right) \geq 2(1+2 \gamma)(1-2 r-a) .
$$

Example 2.2. Notice that the functions $u_{a}^{ \pm}$considered in the previous example agree on the plane $x_{3}=0$, as

$$
u_{a}^{+}\left(x_{1}, x_{2}, 0\right)=u_{a}^{-}\left(x_{1}, x_{2}, 0\right)=\frac{\left(x_{1}, x_{2},-a\right)}{\left|\left(x_{1}, x_{2},-a\right)\right|} .
$$

Hence we may define another couple of functions $\left(\widetilde{u}_{a}^{+}, \widetilde{u}_{a}^{-}\right)$in $W^{1,2}\left(B^{3}, \mathbb{S}^{2}\right)$ which will have different topological properties: set

$$
\widetilde{u}_{a}^{+}(x):=\left\{\begin{array}{ll}
u_{a}^{+}(x) \text { if } & x_{3} \geq 0 \\
u_{a}^{-}(x) \text { if } & x_{3} \leq 0
\end{array} \quad \widetilde{u}_{a}^{-}(x):= \begin{cases}u_{a}^{-}(x) \text { if } & x_{3} \geq 0 \\
u_{a}^{+}(x) \text { if } & x_{3} \leq 0\end{cases}\right.
$$

or, equivalently,

$$
\begin{gathered}
\widetilde{u}_{a}^{+}(x):=\frac{\left(x_{1}, x_{2},\left|x_{3}\right|-a\right)}{\left|\left(x_{1}, x_{2},\left|x_{3}\right|-a\right)\right|}=u_{a}^{+}\left(x_{1}, x_{2},\left|x_{3}\right|\right) \\
\widetilde{u}_{a}^{-}(x):=\frac{\left(x_{1}, x_{2},-\left|x_{3}\right|-a\right)}{\left|\left(x_{1}, x_{2},-\left|x_{3}\right|-a\right)\right|}=u_{a}^{-}\left(x_{1}, x_{2},\left|x_{3}\right|\right) .
\end{gathered}
$$

Notice that for $a>0$ the function $\widetilde{u}_{a}^{-}$is smooth and the function $\widetilde{u}_{a}^{+}$has two point singularities at $P_{a}^{ \pm}$of degree \pm 1 , respectively, whereas for $a=0$ both the functions $\widetilde{u}_{0}^{ \pm}$have one point singularity at the origin of degree zero.

In the case $a=0$, for the function $\widetilde{u}_{0}^{ \pm}$we may choose $L_{\widetilde{u}_{0}^{+}}=L_{\widetilde{u}_{0}^{-}}=0$, so that

$$
\mathcal{G}_{\gamma}\left(\widetilde{u}_{0}^{+}, \widetilde{u}_{0}^{-}\right)=0
$$

and therefore

$$
\widetilde{\mathcal{E}_{\gamma}}\left(u_{0}^{+}, u_{0}^{-}\right)=\mathcal{E}_{\gamma}\left(u_{0}^{+}, u_{0}^{-}\right) .
$$

Analogously, in the case $2 a<1-2 r$, we take $L_{\widetilde{u}_{a}^{-}}=0$, and as $L_{\widetilde{u}_{a}^{+}}$we may choose the segment joining $P_{a}^{+}$ and $P_{a}^{-}$, so that

$$
\mathcal{G}_{\gamma}\left(\widetilde{u}_{a}^{+}, \widetilde{u}_{a}^{+}\right)=0+(1+2 \gamma) 2 a \text {. }
$$

\section{The case of multifunctions}

As explained in the Introduction, when dealing with multifunctions we shall identify different couples $(u, v)$ and $(\widetilde{u}, \widetilde{v})$ of Sobolev functions in $W^{1,2}\left(B^{3}, \mathbb{S}^{2}\right)$ provided that the set of points $x$ in the domain $B^{3}$ such that the sets $\{u(x), v(x)\}$ and $\{\widetilde{u}(x), \widetilde{v}(x)\}$ are different has measure zero. The energy of a multifunction is defined by the expression (1.6). A natural convergence is attached to equivalence classes of couples (see Def. 8.3), and we are interested in finding an explicit formula for the relaxed functional of the energy (1.6) with respect to the topology induced by such a convergence (see the definition in formula (8.5)). 
However, we are not able to find an explicit formula for (8.5). As a consequence, we argue in a different way, that is suggested by a relaxation result for equivalence classes of currents (Prop. 8.6) and we shall introduce in (8.12) a suitable relaxed energy $\widetilde{E_{\gamma}}[u, v]$. Again for $\gamma>\min \left\{\gamma_{s}(r), \gamma_{c}(r)\right\}$, we shall obtain that

$$
\widetilde{E_{\gamma}}(u, v)=\inf _{(\widetilde{u}, \widetilde{v})}\left[\mathcal{E}_{\gamma}(\widetilde{u}, \widetilde{v})+\mathcal{G}_{\gamma}(\widetilde{u}, \widetilde{v})\right],
$$

where the infimum is taken among all $(\widetilde{u}, \widetilde{v})$ equivalent to $(u, v)$, and the non-negative functional $\mathcal{G}_{\gamma}$ is defined exactly as in (2.1).

Example 2.3. Going back to Examples 2.1 and 2.2, it is immediate to realize that the couple $\left(\widetilde{u}_{a}^{+}, \widetilde{u}_{a}^{-}\right)$is equivalent to $\left(u_{a}^{+}, u_{a}^{-}\right)$according to the equivalence relation we have just defined. Moreover, the pull-back of a point $y \in \mathbb{S}^{2}$ by the maps $u_{a}^{ \pm}$is an open line segment $L^{ \pm}(a, y)$ with an end point in $P_{a}^{ \pm}$and the other in the boundary of $B^{3}$, and $L^{-}(a, y)$ is obtained from $L^{+}(a, y)$ by reflection with respect to the plane $x_{3}=0$. This yields that the equivalence class $\left[u_{a}^{+}, u_{a}^{-}\right]$contains only two (unordered) couples of representatives, namely $\left(u_{a}^{+}, u_{a}^{-}\right)$and $\left(\widetilde{u}_{a}^{+}, \widetilde{u}_{a}^{-}\right)$. In particular, we have

$$
E_{\gamma}\left[u_{a}^{+}, u_{a}^{-}\right]=\min \left\{\mathcal{E}_{\gamma}\left(u_{a}^{+}, u_{a}^{-}\right), \mathcal{E}_{\gamma}\left(\widetilde{u}_{a}^{+}, \widetilde{u}_{a}^{-}\right)\right\} .
$$

Example 2.4. We see that, in Example 2.3, the choice of the representative $\left(\widetilde{u}_{a}^{+}, \widetilde{u}_{a}^{-}\right)$is favorable in the formula (2.2) for the relaxed energy. More precisely, we choose $0 \leq a<1 / 4$ and $0<r<1 / 4-a$, so that the difference between the gap terms is uniformly estimated by

$$
\mathcal{G}_{\gamma}\left(u_{a}^{+}, u_{a}^{+}\right)-\mathcal{G}_{\gamma}\left(\widetilde{u}_{a}^{+}, \widetilde{u}_{a}^{+}\right) \geq 1+2 \gamma .
$$

Moreover, both the energies $\mathcal{E}_{\gamma}\left(u_{a}^{+}, u_{a}^{-}\right)$and $\mathcal{E}_{\gamma}\left(\widetilde{u}_{a}^{+}, \widetilde{u}_{a}^{-}\right)$converge as $r \rightarrow 0$ to the pointwise interaction energy $\mathcal{E}_{\gamma}^{0}\left(u_{a}^{+}, u_{a}^{-}\right)$, see (1.7). Therefore, we find $0<r_{a}<1 / 4-a$ such that for every radius $0<r<r_{a}$ we also have

$$
\left|\mathcal{E}_{\gamma}\left(u_{a}^{+}, u_{a}^{-}\right)-\mathcal{E}_{\gamma}\left(\widetilde{u}_{a}^{+}, \widetilde{u}_{a}^{-}\right)\right|<\frac{1+2 \gamma}{2} .
$$

This clearly gives

$$
\mathcal{E}_{\gamma}\left(\widetilde{u}_{a}^{+}, \widetilde{u}_{a}^{-}\right)+\mathcal{G}_{\gamma}\left(\widetilde{u}_{a}^{+}, \widetilde{u}_{a}^{+}\right)<\mathcal{E}_{\gamma}\left(u_{a}^{+}, u_{a}^{-}\right)+\mathcal{G}_{\gamma}\left(u_{a}^{+}, u_{a}^{+}\right)-\frac{1+2 \gamma}{2}
$$

and finally that

$$
\widetilde{E_{\gamma}}\left[\widetilde{u}_{a}^{+}, \widetilde{u}_{a}^{-}\right]=\mathcal{E}_{\gamma}\left(\widetilde{u}_{a}^{+}, \widetilde{u}_{a}^{-}\right)+\mathcal{G}_{\gamma}\left(\widetilde{u}_{a}^{+}, \widetilde{u}_{a}^{+}\right)=\mathcal{E}_{\gamma}\left(\widetilde{u}_{a}^{+}, \widetilde{u}_{a}^{-}\right)+2(1+2 \gamma) a .
$$

\section{Cartesian currents and Dirichlet energy}

Let $n \geq 2$. In the sequel we shall denote by $\pi: B^{n} \times \mathbb{R}^{3} \rightarrow B^{n}$ and $\widehat{\pi}: B^{n} \times \mathbb{R}^{3} \rightarrow \mathbb{R}^{3}$ the orthogonal projections onto the first and second factor. Moreover, $\mathcal{H}^{k}$ denotes the $k$-dimensional Hausdorff measure. We refer to [4] and [12] for the general notation on Geometric Measure Theory.

\section{Currents carried by graphs}

Following [7] (see also [9]) if $u: B^{n} \rightarrow \mathbb{S}^{2}$ is a smooth map, the $n$-current $G_{u}$ is defined by the integration of compactly supported smooth $n$-forms $\omega$ in $B^{n} \times \mathbb{S}^{2}$ over the naturally oriented $n$-manifold given by the graph $\mathcal{G}_{u}$ of $u$, i.e.,

$$
G_{u}(\omega):=\int_{\mathcal{G}_{u}} \omega, \quad \omega \in \mathcal{D}^{n}\left(B^{n} \times \mathbb{S}^{2}\right)
$$

Writing explicitly the action of $\omega$ on $\mathcal{G}_{u}$ in terms of the pull-back via the graph map $(\operatorname{Id} \bowtie u)(x):=(x, u(x))$, the above definition extends to the more general class of Sobolev maps in $W^{1,2}\left(B^{n}, \mathbb{S}^{2}\right)$ (defined in (1.2)). It 
turns out that for every $u \in W^{1,2}\left(B^{n}, \mathbb{S}^{2}\right)$, the current $G_{u}$ is an integer multiplicity (say i.m.) rectifiable current in $\mathcal{R}_{n}\left(B^{n} \times \mathbb{S}^{2}\right)$, the action on forms being given in a weak sense by

$$
G_{u}(\omega)=\int_{B^{n}}(\operatorname{Id} \bowtie u)^{\#} \omega \quad \forall \omega \in \mathcal{D}^{n}\left(B^{n} \times \mathbb{S}^{2}\right) .
$$

This time, the $n$-rectifiable graph $\mathcal{G}_{u}$ is the subset of $B^{n} \times \mathbb{S}^{2}$ given by the points $(x, u(x))$, where $x$ is a Lebesgue point of both $u$ and $D u$ and $u(x)$ is the Lebesgue value of $u$.

Moreover, by the area formula the $\mathcal{H}^{n}$-measure of $\mathcal{G}_{u}$ agrees with the area:

$$
A\left(u, B^{n}\right):=\int_{B^{n}} J_{n}(\mathrm{Id} \bowtie u) \mathrm{d} x=\mathcal{H}^{n}\left(\mathcal{G}_{u}\right),
$$

where $J_{n}(\operatorname{Id} \bowtie u)$ is the $n$-dimensional Jacobian of the mapping $\operatorname{Id} \bowtie u$.

Since moreover $u$ takes values into $\mathbb{S}^{2}$, the parallelogram inequality yields

$$
J_{n}(\operatorname{Id} \bowtie u) \leq c_{n}\left(1+|D u|^{2}\right)
$$

for some dimensional constant $c_{n}>0$. Therefore, by the area formula, $G_{u}$ has finite mass

$$
\mathbf{M}\left(G_{u}\right)=\int_{B^{n}} J_{n}(\operatorname{Id} \bowtie u) \mathrm{d} x \leq c_{n} \int_{B^{n}}\left(1+|D u|^{2}\right) \mathrm{d} x<\infty
$$

and the mass $\mathbf{M}\left(G_{u}\right)$ agrees with the area $A\left(u, B^{n}\right)$, see (3.2).

\section{Boundary}

If $u \in W^{1,2}\left(B^{n}, \mathbb{S}^{2}\right)$ is smooth, by Stokes' theorem the current $G_{u}$ has null boundary inside $B^{n} \times \mathbb{S}^{2}$, as for every compactly supported smooth $(n-1)$-form $\omega$ in $B^{n} \times \mathbb{S}^{2}$

$$
\partial G_{u}(\omega):=G_{u}(\mathrm{~d} \omega)=\int_{\mathcal{G}_{u}} \mathrm{~d} \omega=\int_{\partial \mathcal{G}_{u}} \omega=0
$$

In dimension $n=2$, thanks to Schoen-Uhlenbeck density theorem [11], one obtains that the null-boundary condition

$$
\partial G_{u}(\omega)=G_{u}(\mathrm{~d} \omega)=0 \quad \forall \omega \in \mathcal{D}^{n-1}\left(B^{n} \times \mathbb{S}^{2}\right)
$$

is always satisfied for every $u \in W^{1,2}\left(B^{2}, \mathbb{S}^{2}\right)$. Also, if $n \geq 3$, condition (3.3) holds true if e.g. $u \in W^{1, n}\left(B^{n}, \mathbb{S}^{2}\right)$, or if $u$ is Lipschitz continuous. However, in general (3.3) is violated in $W^{1,2}\left(B^{n}, \mathbb{S}^{2}\right)$, as the following example shows.

Example 3.1. If $n=3$ and $u(x):=x /|x|$, we have $u \in W^{1,2}\left(B^{3}, \mathbb{S}^{2}\right)$ but

$$
\partial G_{u}=-\delta_{0} \times \llbracket \mathbb{S}^{2} \rrbracket \quad \text { on } \mathcal{D}^{2}\left(B^{3} \times \mathbb{S}^{2}\right)
$$

where $\delta_{0}$ is the unit Dirac mass at the origin, compare [7], Vol. I, Section 3.2.2.

\section{Cartesian currents}

We now introduce the class of Cartesian currents that will be relevant in our analysis.

Definition 3.2. The class of Cartesian currents $\operatorname{cart}^{2,1}\left(B^{n} \times \mathbb{S}^{2}\right)$ is given by all the i.m. rectifiable currents $T \in \mathcal{R}_{n}\left(B^{n} \times \mathbb{S}^{2}\right)$ with finite mass, $\mathbf{M}(T)<\infty$, with null boundary in $B^{n} \times \mathbb{S}^{2}$,

$$
\partial T(\omega):=T(\mathrm{~d} \omega)=0 \quad \forall \omega \in \mathcal{D}^{n-1}\left(B^{n} \times \mathbb{S}^{2}\right),
$$

that can be decomposed as

$$
T=G_{u_{T}}+L_{T} \times \llbracket \mathbb{S}^{2} \rrbracket
$$

for some i.m. rectifiable current $L_{T} \in \mathcal{R}_{n-2}\left(B^{n}\right)$ and some Sobolev map $u_{T} \in W^{1,2}\left(B^{n}, \mathbb{S}^{2}\right)$. 
Notice that the mass of a current $T$ in $\operatorname{cart}^{2,1}\left(B^{n} \times \mathbb{S}^{2}\right)$ on the cylinder $B \times \mathbb{S}^{2}$, where $B \subset B^{n}$ is any Borel set, is given by

$$
\mathbf{M}\left(T\left\llcorner B \times \mathbb{S}^{2}\right)=\int_{B} J_{n}\left(\operatorname{Id} \bowtie u_{T}\right) \mathrm{d} x+4 \pi \mathbf{M}\left(L_{T}\llcorner B)<\infty .\right.\right.
$$

We also recall that the weak convergence $T_{k} \rightarrow T$ in the class of currents in $\mathcal{D}_{n}\left(B^{n} \times \mathbb{S}^{2}\right)$ is defined in the dual sense by

$$
T_{k}(\omega) \rightarrow T(\omega) \quad \forall \omega \in \mathcal{D}^{n}\left(B^{n} \times \mathbb{S}^{2}\right),
$$

and that the mass is lower semicontinuous with respect to the weak convergence. Finally, observe that the null boundary condition (3.4) is preserved by the weak convergence.

As a consequence, by Federer-Fleming's closure theorem [5], the weak limit points of sequences of currents $G_{u_{k}}$ carried by the graph of smooth maps $u_{k}: B^{n} \rightarrow \mathbb{S}^{2}$ with equibounded Dirichlet energies, $\sup _{k} \mathbf{D}\left(u_{k}\right)<\infty$, belong to the class $\operatorname{cart}^{2,1}\left(B^{n} \times \mathbb{S}^{2}\right)$.

Remark 3.3. Notice that if $G_{u_{k}} \rightarrow T$ as currents, then $u_{k}$ weakly converges in $W^{1,2}$ to the Sobolev map $u_{T}$ corresponding to $T \in \operatorname{cart}^{2,1}\left(B^{n} \times \mathbb{S}^{2}\right)$. Moreover, in dimension $n \geq 3$, since in general $u_{T}$ does not satisfy the null-boundary condition (3.3), the corresponding current $L_{T} \in \mathcal{R}_{n-2}\left(B^{n}\right)$ in the decomposition (3.5) is non zero and has a boundary with possibly infinite mass, $\mathbf{M}\left(\left(\partial L_{T}\right)\left\llcorner B^{n}\right) \leq+\infty\right.$.

For future use, in dimension $n \geq 3$, for any $u \in W^{1,2}\left(B^{n}, \mathbb{S}^{2}\right)$ we denote by

$$
\mathcal{T}_{u}:=\left\{T \in \operatorname{cart}^{2,1}\left(B^{n} \times \mathbb{S}^{2}\right) \mid u_{T}=u \text { in }(3.5)\right\}
$$

the class of Cartesian currents with corresponding $W^{1,2}$-function equal to $u$.

Remark 3.4. Using the argument in [1] and the density result from [2], it turns out that the class $\mathcal{T}_{u}$ is non-empty for every $u \in W^{1,2}\left(B^{n}, \mathbb{S}^{2}\right)$, compare also [9], Section 5.6. Moreover, for a current $T \in \mathcal{T}_{u}$, the null-boundary condition (3.4) can be equivalently written as $\left(\partial L_{T}\right)\left\llcorner B^{n}=-\mathbb{P}(u)\right.$, where $\mathbb{P}(u) \in \mathcal{D}_{n-3}\left(B^{n}\right)$ is a suitable $(n-3)$-current that describes the relevant singularities of $u$.

Example 3.5. If $n=3$ and $T \in \mathcal{T}_{u}$ with $u(x)=x /|x|$, see Example 3.1, we have that $\mathbb{P}(u)=-\delta_{0}$. Therefore, we deduce that $L_{T}$ is any 1 -current in $\mathcal{R}_{1}\left(B^{3}\right)$ satisfying $\left(\partial L_{T}\right)\left\llcorner B^{3}=\delta_{0}\right.$.

\section{The Dirichlet energy on currents}

According to (3.6), for any Borel set $B \subset B^{n}$ denote by

$$
\mathbf{D}\left(T, B \times \mathbb{S}^{2}\right):=\mathbf{D}\left(u_{T}, B\right)+4 \pi \mathbf{M}\left(L_{T}\llcorner B)\right.
$$

the Dirichlet energy of a current $T$ in $\operatorname{cart}^{2,1}\left(B^{n} \times \mathbb{S}^{2}\right)$ in $B \times \mathbb{S}^{2}$, where we have set

$$
\mathbf{D}(u, B):=\frac{1}{2} \int_{B}|D u|^{2} \mathrm{~d} x, \quad u \in W^{1,2}\left(B^{n}, \mathbb{S}^{2}\right) ;
$$

for simplicity we introduce the notation

$$
\mathbf{D}(T):=\mathbf{D}\left(T, B^{n} \times \mathbb{S}^{2}\right)=\mathbf{D}\left(u_{T}\right)+4 \pi \mathbf{M}\left(L_{T}\right) .
$$

We next consider the parametric polyconvex l.s.c. extension $F: \mathbb{R}^{3} \times \Lambda_{n} \mathbb{R}^{n+3} \rightarrow[0,+\infty]$ of the Dirichlet integrand for mappings from $B^{n}$ into $\mathbb{S}^{2}$, as defined in [7], Vol. II, Section 1.2.4, Proposition 12, see also [9], Section 4.9. It turns out that for every Borel set $B \subset B^{n}$

$$
\mathbf{D}\left(T, B \times \mathbb{S}^{2}\right)=\int_{B \times \mathbb{S}^{2}} F(\widehat{\pi}(z), \vec{T}(z)) \mathrm{d}\|T\|(z)
$$


where $\vec{T}$ is the Radon-Nikodym derivative of $T$ with respect to the mass density $\|T\|$, so that

$$
T(\omega)=\int_{B^{n} \times \mathbb{S}^{2}}\langle\vec{T}, \omega\rangle \mathrm{d}\|T\| \quad \forall \omega \in \mathcal{D}^{n}\left(B^{n} \times \mathbb{S}^{2}\right) .
$$

This implies that the functional $T \mapsto \mathbf{D}(T)$ is lower semicontinuous with respect to the weak convergence in $\mathcal{D}_{n}\left(B^{n} \times \mathbb{S}^{2}\right)$ along sequences of currents in $\operatorname{cart}^{2,1}\left(B^{n} \times \mathbb{S}^{2}\right)$.

The following property of future use can be obtained by using arguments similar to the ones after Definition 3 in [7], Vol. II, Section 1.2.4, see also [9], Section 4.8.

Lemma 3.6. Let $F: \mathbb{R}^{3} \times \Lambda_{n} \mathbb{R}^{n+3} \rightarrow[0,+\infty]$ be the parametric polyconvex l.s.c. extension of the Dirichlet integrand as above. Then for every non-negative lower semicontinuous function $a: B^{n} \rightarrow[0,+\infty)$ the functional

$$
T \mapsto \int_{B^{n} \times \mathbb{S}^{2}} a(\pi(z)) F(\widehat{\pi}(z), \vec{T}(z)) \mathrm{d}\|T\|(z)
$$

is lower semicontinuous with respect to the weak convergence in $\mathcal{D}_{n}\left(B^{n} \times \mathbb{S}^{2}\right)$ along sequences of currents in $\operatorname{cart}^{2,1}\left(B^{n} \times \mathbb{S}^{2}\right)$.

Remark 3.7. We shall use the standard notation $\mathbb{I}_{B}$ for the characteristic function of a subset $B \subset B^{n}$, i.e. we set

$$
\mathbb{I}_{B}(x):= \begin{cases}1 \text { if } & x \in B \\ 0 \text { if } & x \notin B .\end{cases}
$$

Applying Lemma 3.6 to the particular case where $a(x)=\mathbb{I}_{B}(x)$ for some open set $B$ in $B^{n}$ we deduce the lower semicontinuity of $T \mapsto \mathbf{D}\left(T, B \times \mathbb{S}^{2}\right)$.

The following closure property holds, see [6]:

Proposition 3.8. The class cart ${ }^{2,1}\left(B^{n} \times \mathbb{S}^{2}\right)$ is sequentially weakly closed in $\mathcal{D}_{n}\left(B^{n} \times \mathbb{S}^{2}\right)$ along sequences with equibounded Dirichlet energies, $\sup _{k} \mathbf{D}\left(T_{k}\right)<\infty$, and contains the weak limits of sequences of graphs of smooth maps in $W^{1,2}\left(B^{n}, \mathbb{S}^{2}\right)$ with equibounded Dirichlet integrals, $\sup _{k} \mathbf{D}\left(u_{k}\right)<\infty$.

We finally recall the following strong density result, proved by Giaquinta-Modica-Souček in 1989, in the cases $n=2$ and $n=3$, see Sections 4.1.2 and 4.2.5 in [7], Vol. II, and in [8] in higher dimension $n \geq 4$, see also [9], Chapter 5 .

Theorem 3.9. For every $T \in \operatorname{cart}^{2,1}\left(B^{n} \times \mathbb{S}^{2}\right)$, there exists a sequence $\left\{u_{k}\right\} \subset C^{\infty}\left(B^{n}, \mathbb{S}^{2}\right)$ of smooth maps such that $G_{u_{k}} \rightarrow T$ weakly in $\mathcal{D}_{n}\left(B^{n} \times \mathbb{S}^{2}\right)$ and $\mathbf{D}\left(u_{k}\right) \rightarrow \mathbf{D}(T)$, as $k \rightarrow \infty$.

\section{The relaxed Dirichlet energy}

Consider the relaxed energy of maps $u \in W^{1,2}\left(B^{n}, \mathbb{S}^{2}\right)$

$$
\widetilde{\mathbf{D}}(u):=\inf \left\{\liminf _{k \rightarrow \infty} \mathbf{D}\left(u_{k}\right) \mid\left\{u_{k}\right\} \subset C^{\infty}\left(B^{n}, \mathbb{S}^{2}\right), u_{k} \rightarrow u \text { weakly in } W^{1,2}\left(B^{n}, \mathbb{R}^{3}\right)\right\} .
$$

Using Theorem 3.9 and the lower semicontinuity of the Dirichlet energy $T \mapsto \mathbf{D}(T)$ in $\operatorname{cart}^{2,1}\left(B^{n} \times \mathbb{S}^{2}\right)$, one obtains the following representation formula, first proved in [13] for higher dimension $n \geq 4$ :

Proposition 3.10. Let $n \geq 3$. For every $u \in W^{1,2}\left(B^{n}, \mathbb{S}^{2}\right)$ the relaxed energy $\widetilde{\mathbf{D}}(u)$ is finite and there holds

$$
\widetilde{\mathbf{D}}(u)=\inf \left\{\mathbf{D}(T) \mid T \in \mathcal{T}_{u}\right\} .
$$


Remark 3.11. We deduce that the gap between the energy and the relaxed energy amounts to the area of $\mathbb{S}^{2}$ times $4 \pi \mathbf{M}\left(L_{u}\right)$, where $L_{u} \in \mathcal{R}_{n-2}\left(B^{n}\right)$ is mass minimizing among all the i.m. rectifiable $(n-2)$ currents $L$ in $B^{n}$ such that $(\partial L)\left\llcorner B^{n}=\mathbb{P}(u)\right.$. (This means that $L_{u}$ is the integral minimal connection of the current $\mathbb{P}(u)$ in $\mathcal{D}_{n-3}\left(B^{n}\right)$ of the singularity of $u$, see also Rem. 3.4). Finally, in the case of dimension $n=2$, by Schoen-Uhlenbeck density theorem one clearly has

$$
\widetilde{\mathbf{D}}(u)=\mathbf{D}(u) \quad \forall u \in W^{1,2}\left(B^{2}, \mathbb{S}^{2}\right) .
$$

\section{A LOWER SEMiCONTINUOUS FUnCTIONAL ON CURRENTS}

In order to properly extend the energy (1.4) to couples of Cartesian currents, we preliminarily rewrite it as

$$
\mathcal{E}_{\gamma}(u, v):=(1+\gamma)(\mathbf{D}(u)+\mathbf{D}(v))+\gamma(\Phi(u)+\Phi(v))-\gamma(C(u, v)+C(v, u)),
$$

where $\mathbf{D}(u)$ and $\mathbf{D}(v)$ are the standard Dirichlet integrals (see (1.1)), the non-local term is

$$
\Phi(w):=\int_{B^{n}} f_{\widetilde{B}_{r}(x)} \frac{1}{2}|D w(y)|^{2} \mathrm{~d} y \mathrm{~d} x
$$

and the continuous term (such terminology comes from Step 1 in the proof of Thm. 4.2) is

$$
C\left(w_{1}, w_{2}\right):=\int_{B^{n}} f_{\widetilde{B}_{r}(x)}\left(D w_{1}(x) \bullet D w_{2}(y)\right) \mathrm{d} y \mathrm{~d} x,
$$

where $A \bullet B:=\operatorname{trace}\left(A B^{T}\right)$, for $(3 \times n)$-matrices $A, B$.

Now, let $T_{1}, T_{2}$ be Cartesian currents in $\operatorname{cart}^{2,1}\left(B^{n} \times \mathbb{S}^{2}\right)$ and according to Definition 3.2 write

$$
T_{i}=G_{u_{i}}+L_{i} \times \llbracket \mathbb{S}^{2} \rrbracket, \quad i=1,2,
$$

so that $u_{i}=u_{T_{i}} \in W^{1,2}\left(B^{n}, \mathbb{S}^{2}\right)$ and $L_{i}=L_{T_{i}} \in \mathcal{R}_{n-2}\left(B^{n}\right)$. For every $\gamma \in \mathbb{R}$ we introduce the energy functional

$$
\mathcal{E}_{\gamma}\left(T_{1}, T_{2}\right):=\mathcal{E}_{\gamma}\left(u_{1}, u_{2}\right)+4 \pi(1+\gamma)\left(\mathbf{M}\left(L_{1}\right)+\mathbf{M}\left(L_{2}\right)\right)+4 \pi \gamma\left(\Psi\left(L_{1}\right)+\Psi\left(L_{2}\right)\right),
$$

and we notice that $\mathcal{E}_{\gamma}\left(T_{2}, T_{1}\right)=\mathcal{E}_{\gamma}\left(T_{1}, T_{2}\right)$. In (4.4), according to (4.2), we have set

$$
\Psi(L):=\int_{B^{n}}\left|\widetilde{B}_{r}(x)\right|^{-1} \mathbf{M}\left(L\left\llcorner\widetilde{B}_{r}(x)\right) \mathrm{d} x, \quad L \in \mathcal{R}_{n-2}\left(B^{n}\right) .\right.
$$

Recalling (4.1) and the expression of the extension of the Dirichlet energy to currents in (3.8), and comparing (4.5) with (4.2), we see that the functional $\mathcal{E}_{\gamma}\left(T_{1}, T_{2}\right)$ in (4.4) is the natural extension to couples of currents in cart $^{2,1}$ of the functional $\mathcal{E}_{\gamma}\left(u_{1}, u_{2}\right)$ defined in (4.1).

The goal of this section is to show the lower semicontinuity of the functional $\mathcal{E}_{\gamma}\left(T_{1}, T_{2}\right)$ with respect to weak convergence in $\mathcal{D}_{n}\left(B^{n} \times \mathbb{S}^{2}\right)$ along sequences with equibounded Dirichlet energy. We are able to achieve this for any $\gamma$ greater than a threshold $\gamma_{s}(r)<0$ given by

$$
\gamma_{s}(r):=\frac{1}{c(r) r^{n}+1}-1
$$

where

$$
c(r):=\inf _{x \in B^{n}} \frac{\left|\widetilde{B}_{r}(x)\right|}{\left|B_{r}\right|}>0 .
$$


Remark 4.1. From simple geometric arguments we find that

$$
c(r)\left|B_{r}\right| \leq\left|\widetilde{B}_{r}(x)\right| \leq\left|B_{r}\right|, \quad 2^{-n} \leq c(r) \leq 1 / 2 .
$$

These estimates depend on the choice $\Omega=B^{n}$ but only the constants change in the case of a Lipschitz set $\Omega \subset \mathbb{R}^{n}$, yielding $0<\alpha(\Omega) \leq c(r) \leq 1$. In particular $\gamma_{s}(r)$ depends on $\Omega$ and $r$ in the general case.

The main result of this section is the following theorem, in which we prove lower semicontinuity for the functional $\mathcal{E}_{\gamma}\left(T_{1}, T_{2}\right)$ in the case when $\gamma \geq \gamma_{s}(r)$.

Theorem 4.2. For every $\gamma \geq \gamma_{s}(r)$ the functional $\mathcal{E}_{\gamma}\left(T_{1}, T_{2}\right)$ defined in (4.4) is lower semicontinuous along couples of sequences of currents in cart $^{2,1}\left(B^{n} \times \mathbb{S}^{2}\right)$ with equibounded Dirichlet energies.

Remark 4.3. Notice that $\gamma_{s}(r)<0$ : the strategy of our proof in the case $\gamma_{s}(r) \leq \gamma<0$ is different from the one in the case $\gamma>0$. Some remarks on the lack of sharpness of this threshold will be made in Section 7 .

In the course of the proof of Theorem 4.2 we will need the following technical lemma.

Lemma 4.4. Let $r>0$ and $L \subset B^{n}$ be such that $\mathcal{H}^{n-2}(L)<\infty$. Then there exists a set $C \subset B^{n}$ with $\mathcal{L}^{n}(C)=0$ such that

$$
\mathcal{H}^{n-2}\left(\partial B_{r}\left(x_{0}\right) \cap L\right)=0
$$

for all $x_{0} \in B^{n} \backslash C$; in the case $n \geq 3, C$ is at most countable.

Proof. The case $n=2$ is trivial: $C$ consists of a finite number of circles, so we just treat the case $n \geq 3$. An easy geometrical remark we use in the proof is the following: if $x, y, z$ are three distinct points in $\mathbb{R}^{n}$ with $n \geq 3$ then

$$
\mathcal{H}^{n-2}\left(\partial B_{r}(x) \cap \partial B_{r}(y) \cap \partial B_{r}(z)\right)=0 .
$$

This is obvious if the three are aligned; if not, project on the 2-plane through the centers the intersection $\partial B_{r}(x) \cap \partial B_{r}(y)$ and the intersection $\partial B_{r}(y) \cap \partial B_{r}(z)$. In the worst case these will be nonparallel segments which intersect in a point, thus $\partial B_{r}(x) \cap \partial B_{r}(y) \cap \partial B_{r}(z)$ is contained in an $(n-2)$-plane, but the intersection of $\partial B_{r}(x)$ with any such plane has zero $(n-2)$-dimensional measure.

Thus, it is impossible that for any given $x, y, z$ we have

$$
\mathcal{H}^{n-2}\left(L \cap \partial B_{r}(x) \cap \partial B_{r}(y) \cap \partial B_{r}(z)\right)>0 .
$$

We do not have to take care of three overlapping intersections of balls with $L$. Now we deal with two overlapping intersections: set

$$
C_{2}=\left\{x \in \mathbb{R}^{n}: \exists y \in \mathbb{R}^{n}: \mathcal{H}^{n-2}\left(L \cap \partial B_{r}(x) \cap \partial B_{r}(y)\right)>0\right\}
$$

and call

$$
\mathcal{F}_{2}=\left\{M \subset L: \mathcal{H}^{n-2}(M)>0, \exists x, y \in C_{2}: M=L \cap \partial B_{r}(x) \cap \partial B_{r}(y)\right\}
$$

the set of intersections. For any $M \in \mathcal{F}_{2}$, the centers $x, y$ such that $M=L \cap \partial B_{r}(x) \cap \partial B_{r}(y)$ are unique by (4.8). Moreover, again by (4.8), if $M, M^{\prime} \in \mathcal{F}_{2}$ are distinct then

$$
\mathcal{H}^{n-2}\left(M \cap M^{\prime}\right)=0
$$

so the sets in $\mathcal{F}_{2}$ are essentially disjoint, have positive $\mathcal{H}^{n-2}$ measure and are contained in $L$ which has finite $\mathcal{H}^{n-2}$ measure and therefore $\mathcal{F}_{2}$ is at most countable, and so is $C_{2}$ by the 1-1 relationship between sets $M$ and centers $x, y$. 
We now shave off $L$ all the sets $\partial B_{r}(x)$ with $x$ in $C_{2}$; remark we are not only subtracting the sets in $\mathcal{F}_{2}$ but maybe some larger chunks:

$$
L_{1}=L \backslash\left(\bigcup_{x \in C_{2}} \partial B_{r}(x)\right) .
$$

Set

$$
C_{1}=\left\{x \in \mathbb{R}^{n}: \mathcal{H}^{n-2}\left(L_{1} \cap \partial B_{r}(x)\right)>0\right\}
$$

and call

$$
\mathcal{F}_{1}=\left\{M \subset L_{1}: \mathcal{H}^{n-2}(M)>0, \exists x \in C_{1}: M=L_{1} \cap \partial B_{r}(x)\right\} .
$$

We remark that if $x \in C_{1}$ then $x \notin C_{2}$ by the definition of $L_{1}$. Also, if $x \in C_{1}$ then

$$
\mathcal{H}^{n-2}\left(\partial B_{r}(x) \cap\left(L \backslash L_{1}\right)\right)=0
$$

because otherwise $x \in C_{2}$. Finally, if $x, y \in C_{1}$ then

$$
\mathcal{H}^{n-2}\left(L_{1} \cap \partial B_{r}(x) \cap \partial B_{r}(y)\right)=0
$$

and again both $\mathcal{F}_{1}$ and $C_{1}$ are at most countable. But if $x \notin C_{1} \cup C_{2}$ then $\mathcal{H}^{n-2}\left(\partial B_{r}(x) \cap L\right)=0$.

Proof of Theorem 4.2. The proof will be divided into three steps.

Step 1. The continuous term. We first observe that the term (4.3) may be written in components as

$$
C(u, v)=\sum_{i=1}^{n} \sum_{j=1}^{3} \int_{B^{n}} f_{\widetilde{B}_{r}(x)} D_{i} u^{j}(x) D_{i} v^{j}(y) \mathrm{d} y \mathrm{~d} x .
$$

If $D v_{k} \rightarrow D v$ weakly in $L^{2}$, for any $i, j$ the sequence

$$
V_{k}(x)=f_{\widetilde{B}_{r}(x)} D_{i} v_{k}^{j}(y) \mathrm{d} y
$$

is thus bounded in $L^{\infty}$ and converges pointwise, so that we get the strong convergence

$$
V_{k}(x) \rightarrow f_{\widetilde{B}_{r}(x)} D_{i} v^{j}(y) \mathrm{d} y \quad \text { strongly in } L^{2} .
$$

We then deduce that for any sequences $\left\{u_{k}\right\},\left\{v_{k}\right\} \subset W^{1,2}\left(B^{n}, \mathbb{S}^{2}\right)$ such that $u_{k} \rightarrow u$ and $v_{k} \rightarrow v$ weakly in $W^{1,2}\left(B^{n}, \mathbb{R}^{3}\right)$, we have

$$
\lim _{k \rightarrow \infty} C\left(u_{k}, v_{k}\right)=C(u, v) \quad \forall u, v \in W^{1,2}\left(B^{n}, \mathbb{S}^{2}\right) .
$$

Step 2. The case $\gamma \geq 0$. According to the notation from the previous section, we have

$$
\mathcal{E}_{\gamma}\left(T_{1}, T_{2}\right)=(1+\gamma)\left(\mathbf{D}\left(T_{1}\right)+\mathbf{D}\left(T_{2}\right)\right)-\gamma\left(C\left(u_{1}, u_{2}\right)+C\left(u_{2}, u_{1}\right)\right)+\gamma\left(\Phi\left(T_{1}\right)+\Phi\left(T_{2}\right)\right),
$$

where

$$
\Phi(T):=\int_{B^{n}}\left|\widetilde{B}_{r}(x)\right|^{-1} \mathbf{D}\left(T, \widetilde{B}_{r}(x) \times \mathbb{S}^{2}\right) \mathrm{d} x .
$$

We know that the first term in the right hand side of (4.9) is lower semicontinuous (for any $\gamma \geq-1$ ), and the second one has been dealt with in Step 1, since the weak convergence $T_{k} \rightarrow T$, equipped with condition $\sup _{k} \mathbf{D}\left(T_{k}\right)<\infty$, implies the weak $W^{1,2}$-convergence $u_{T_{k}} \rightarrow u_{T}$. As for the third term, if $\left\{T_{k}\right\} \subset \operatorname{cart}^{2,1}\left(B^{n} \times \mathbb{S}^{2}\right)$ 
and $T_{k} \rightarrow T \in \operatorname{cart}^{2,1}\left(B^{n} \times \mathbb{S}^{2}\right)$ weakly in $\mathcal{D}_{n}\left(B^{n} \times \mathbb{S}^{2}\right)$, by Remark 3.7 and Lemma 4.4, the functional $T \mapsto \mathbf{D}\left(T, \widetilde{B}_{r}(x) \times \mathbb{S}^{2}\right)$ is lower semicontinuous for $\mathcal{L}^{n}$-a.e. $x \in B^{n}$. Therefore, by Fatou's lemma, we have

$$
\begin{aligned}
\Phi(T) & \leq \int_{B^{n}} \liminf _{k \rightarrow \infty}\left|\widetilde{B}_{r}(x)\right|^{-1} \mathbf{D}\left(T_{k}, \widetilde{B}_{r}(x) \times \mathbb{S}^{2}\right) \mathrm{d} x \\
& \leq \liminf _{k \rightarrow \infty} \int_{B^{n}}\left|\widetilde{B}_{r}(x)\right|^{-1} \mathbf{D}\left(T_{k}, \widetilde{B}_{r}(x) \times \mathbb{S}^{2}\right) \mathrm{d} x=\liminf _{k \rightarrow \infty} \Phi\left(T_{k}\right),
\end{aligned}
$$

and the theorem follows for any $\gamma \geq 0$.

Step 3. The case $\gamma<0$. On account of Step 1 and (4.9), it suffices to show that the functional

$$
\mathcal{F}_{\gamma}(T):=(1+\gamma) \mathbf{D}(T)+\gamma \Phi(T)
$$

is weakly lower semicontinuous in $\operatorname{cart}^{2,1}\left(B^{n} \times \mathbb{S}^{2}\right)$.

To this purpose, we observe that for any $T \in \operatorname{cart}^{2,1}\left(B^{n} \times \mathbb{S}^{2}\right)$ we have

$$
\mathcal{F}_{\gamma}(T)=\int_{B^{n}}\left(\frac{1+\gamma}{\left|B^{n}\right|} \mathbf{D}(T)+\frac{\gamma}{\left|\widetilde{B}_{r}(x)\right|} \mathbf{D}\left(T, \widetilde{B}_{r}(x) \times \mathbb{S}^{2}\right)\right) \mathrm{d} x
$$

Therefore, setting

$$
a_{x_{0}}(x):=\left(\frac{1+\gamma}{\left|B^{n}\right|}+\frac{\gamma}{\left|\widetilde{B}_{r}\left(x_{0}\right)\right|} \mathbb{I}_{\widetilde{B}_{r}\left(x_{0}\right)}(x)\right)
$$

using (3.9) we have

$$
\mathcal{F}_{\gamma}(T)=\int_{B^{n}} f_{\gamma}(T, x) \mathrm{d} x
$$

where, for $x_{0} \in B^{n}$,

$$
f_{\gamma}\left(T, x_{0}\right):=\int_{B^{n} \times \mathbb{S}^{2}} a_{x_{0}}(\pi(z)) F(\widehat{\pi}(z), \vec{T}(z)) \mathrm{d}\|T\|(z),
$$

and $F: \mathbb{R}^{3} \times \Lambda_{n} \mathbb{R}^{n+3} \rightarrow[0,+\infty]$ is the parametric polyconvex l.s.c. extension of the Dirichlet integrand.

By Fatou's lemma, it suffices to show that $T \mapsto f_{\gamma}\left(T, x_{0}\right)$ is non negative and lower semicontinuous with respect to $T$ for a.e. $x_{0} \in B^{n}$. To this aim, we apply Lemma 3.6. We need to check that, for a.e. $x_{0}$, the function $a_{x_{0}}$ is non negative and lower semicontinuous. On account of (4.7), we have

$$
\left|B^{n}\right| \inf _{x \in B^{n}} a_{x_{0}}(x)=1+\gamma+\frac{\gamma}{c(r) r^{n}}
$$

so that $a_{x_{0}}(x) \geq 0$ for all $x, x_{0} \in B^{n}$ if and only if $\gamma \geq \gamma_{s}(r)$, where $\gamma_{s}(r)$ is given by (4.6). Finally, Lemma 4.4 implies that, for a.e. $x_{0}$, in the formula for $f_{\gamma}\left(T, x_{0}\right)$ we may restrict the integration to the complement of $\partial \widetilde{B}_{r}\left(x_{0}\right)$, so that without loss of generality one may consider in the definition of $a_{x_{0}}$ the interior of $\widetilde{B}_{r}\left(x_{0}\right)$, which implies the lower semicontinuity of $a_{x_{0}}$.

\section{A COERCIVITY PROPERTY OF THE FUNCTIONAL}

In this section we discuss the coercivity property of the functional $\mathcal{E}_{\gamma}(u, v)$ given by formula (4.1). We define for every $y \in B^{n}$

and set

$$
k_{r}(y):=\int_{B^{n}} \frac{1}{\left|\widetilde{B}_{r}(x)\right|} \mathbb{I}_{B_{r}(y)}(x) \mathrm{d} x=\int_{\widetilde{B}_{r}(y)} \frac{1}{\left|\widetilde{B}_{r}(x)\right|} \mathrm{d} x
$$

$$
K(r):=\sup _{y \in B^{n}} k_{r}(y) .
$$

Under such notation, the main theorem of this section is the following. 
Theorem 5.1. Let

$$
\gamma_{c}(r):=-\frac{1}{(1+\sqrt{K(r)})^{2}}
$$

where $K(r)$ is given by (5.2). Then for every $\gamma>\gamma_{c}(r)$ there exists a constant $C=C(\gamma, r)>0$, only depending on $\gamma$ and $r$, such that for every $u, v \in W^{1,2}\left(B^{n}, \mathbb{S}^{2}\right)$ we have

$$
\mathcal{E}_{\gamma}(u, v) \geq C(\mathbf{D}(u)+\mathbf{D}(v)) .
$$

Moreover, $C(\gamma, r) \geq 1$ if $\gamma \geq 0$, and for $\gamma_{c}(r)<\gamma<0$

$$
C(\gamma, r) \geq 1+\gamma(1+\sqrt{K(r)})^{2}
$$

Before entering in the proof of such theorem, we prove a simple lemma involving the quantity $K(r)$ defined in $(5.2)$.

Lemma 5.2. For every non-negative function $f \in L^{1}\left(B^{n}\right)$ we have

$$
\int_{B^{n}} f_{\widetilde{B}_{r}(x)} f(y) \mathrm{d} y \mathrm{~d} x \leq K(r) \int_{B^{n}} f(x) \mathrm{d} x .
$$

Proof. We have

$$
\begin{aligned}
\int_{B^{n}} f_{\widetilde{B}_{r}(x)} f(y) \mathrm{d} y \mathrm{~d} x & =\int_{B^{n}} \int_{B^{n}} f(y) \frac{1}{\left|\widetilde{B}_{r}(x)\right|} \mathbb{I}_{B_{r}(x)}(y) \mathrm{d} y \mathrm{~d} x \\
& =\int_{B^{n}} f(y) \int_{B^{n}} \frac{1}{\left|\widetilde{B}_{r}(x)\right|} \mathbb{I}_{B_{r}(y)}(x) \mathrm{d} x \mathrm{~d} y,
\end{aligned}
$$

so that

$$
\int_{B^{n}} f_{\widetilde{B}_{r}(x)} f(y) \mathrm{d} y \mathrm{~d} x=\int_{B^{n}} f(x) k_{r}(x) \mathrm{d} x
$$

and we obtain the assertion.

Remark 5.3. We give an estimate for $k_{r}$. Recalling the definition (4.7) of $c(r)$ and the estimate in Remark 4.1, for every $y \in B^{n}$ we have

$$
k_{r}(y)=\int_{\widetilde{B}_{r}(y)} \frac{1}{\left|\widetilde{B}_{r}(x)\right|} \mathrm{d} x \leq \frac{1}{c(r)} \int_{\widetilde{B}_{r}(y)} \frac{1}{\left|B_{r}\right|} \mathrm{d} x \leq \frac{1}{c(r)},
$$

and analogously

$$
k_{r}(y)=\int_{\widetilde{B}_{r}(y)} \frac{1}{\left|\widetilde{B}_{r}(x)\right|} \mathrm{d} x \geq \int_{\widetilde{B}_{r}(y)} \frac{1}{\left|B_{r}\right|} \mathrm{d} x \geq c(r),
$$

so that

$$
c(r) \leq k_{r}(y) \leq \frac{1}{c(r)}
$$

This implies in particular the following estimate on the constant $K(r)$ :

$$
K(r) \leq \frac{1}{c(r)}
$$


This estimate is not optimal, though, because the balls such that $\left|\widetilde{B}_{r}(x)\right| \simeq c(r)\left|B_{r}(x)\right|$ are those with $\|x\| \simeq 1$, whereas the function

$$
\|y\| \mapsto \int_{B^{n}} \frac{1}{\left|\widetilde{B}_{r}(x)\right|} \mathbb{I}_{B_{r}(y)} \mathrm{d} x
$$

is decreasing at 1, i.e., the supremum in (5.2) is attained for any $y$ with $\|y\|=\rho$ for some $1-r<\rho<1$. This monotonicity property can be shown by noticing that (from simple geometric arguments) there exists $0<\alpha(r)<1$ with the property that, for every $y \in \mathbb{S}^{n-1}$ and every $\alpha(r)<\lambda<1$, the strict inclusion $\widetilde{B}_{r}(\lambda y) \varsubsetneqq \widetilde{B}_{r}(y)$ holds.

One may also consider the fact that if one replaces $K(r)$ with $c(r)^{-1}$ in (5.3), passing to the limit as $r \rightarrow 0^{+}$in

$$
\int_{B^{n}} f_{\widetilde{B}_{r}(x)} f(y) \mathrm{d} y \mathrm{~d} x \leq \frac{1}{c(r)} \int_{B^{n}} f(x) \mathrm{d} x
$$

and noticing that $c(r) \rightarrow 1 / 2$, we would obtain the silly estimate

$$
\int_{B^{n}} f(x) \mathrm{d} x \leq 2 \int_{B^{n}} f(x) \mathrm{d} x .
$$

Proof of Theorem 5.1. Using (1.4), the assertion trivially follows for $\gamma \geq 0$. Moreover, writing for every $\eta>0$

$$
|D u(x)-D v(y)|^{2}=-\left|\eta D u(x)+\eta^{-1} D v(y)\right|^{2}+\left(\eta^{2}+1\right)|D u(x)|^{2}+\left(\eta^{-2}+1\right)|D v(y)|^{2},
$$

and a similar formula with $u$ and $v$ interchanged, by (1.4) we find that

$$
\begin{aligned}
2 \mathcal{E}_{\gamma}(u, v)= & \left(1+\gamma\left(\eta^{2}+1\right)\right) \int_{B^{n}}\left(|D u(x)|^{2}+|D v(x)|^{2}\right) \mathrm{d} x \\
& -\gamma \int_{B^{n}} f_{\widetilde{B}_{r}(x)}\left(\left|\eta D u(x)+\eta^{-1} D v(y)\right|^{2}+\left|\eta D v(x)+\eta^{-1} D u(y)\right|^{2}\right) \mathrm{d} y \mathrm{~d} x \\
& +\gamma\left(\eta^{-2}+1\right) \int_{B^{n}} f_{\widetilde{B}_{r}(x)}\left(|D u(y)|^{2}+|D v(y)|^{2}\right) \mathrm{d} y \mathrm{~d} x .
\end{aligned}
$$

By Lemma 5.2 we thus obtain that if $\gamma<0$

$$
2 \mathcal{E}_{\gamma}(u, v) \geq\left[1+\gamma\left(\left(\eta^{2}+1\right)+K(r)\left(\eta^{-2}+1\right)\right)\right] \int_{B^{n}}\left(|D u(x)|^{2}+|D v(x)|^{2}\right) \mathrm{d} x .
$$

The function $\eta \mapsto\left(\left(\eta^{2}+1\right)+K(r)\left(\eta^{-2}+1\right)\right)$ attains its minimum for $\eta=K(r)^{1 / 4}$, and the minimum value is $(1+\sqrt{K(r)})^{2}$, so for $\gamma_{c}(r)<\gamma<0$

$$
2 \mathcal{E}_{\gamma}(u, v) \geq\left[1+\gamma(1+\sqrt{K(r)})^{2}\right] \int_{B^{n}}\left(|D u(x)|^{2}+|D v(x)|^{2}\right) \mathrm{d} x
$$

and the assertion follows.

In the general case of a regular open set, Theorem 5.1 still holds, with the same statement, and the definition (5.2) of the constant $K(r)$ needs only a slight change: just replace $B^{n}$ with the new set (if the set has sharp outward cusps, it may well happen that $K(r)=+\infty)$.

Remark 5.4. By Remark 5.3, the threshold $\gamma_{c}(r)$ in our coercivity theorem is estimated by

$$
\gamma_{c}(r) \leq \widehat{\gamma}(r):=-\frac{1}{\left(1+c(r)^{-1 / 2}\right)^{2}}
$$


and $\widehat{\gamma}(r) \rightarrow-(1+\sqrt{2})^{-2}$ as $r \rightarrow 0^{+}$, since $c(r) \rightarrow 1 / 2$. However, the optimal threshold of the coercivity property for the functional with pointwise interaction (i.e., when $r=0$ ) is $-1 / 4$, whereas $-(1+\sqrt{2})^{-2}>-1 / 4$. Indeed, for every $G, H \in \mathbb{R}^{3 \times n}$

$$
\frac{1}{2}|G|^{2}+\frac{1}{2}|H|^{2}+\gamma|G-H|^{2}=\frac{1+4 \gamma}{2}|G|^{2}+\frac{1+4 \gamma}{2}|H|^{2}-\gamma|G+H|^{2},
$$

which gives immediately the coercivity for any $\gamma>-1 / 4$.

\section{A Representation Result FOR the RELAXED ENERGy}

In this section we prove a representation formula for the relaxed energy (1.5).

We first observe that, from an immediate computation, for every $\gamma \in \mathbb{R}$ we can find an absolute constant $\widetilde{C}=\widetilde{C}(r, \gamma, n)$ such that for every $u, v \in W^{1,2}\left(B^{n}, \mathbb{S}^{2}\right)$ we have

$$
\left|\mathcal{E}_{\gamma}(u, v)\right| \leq \widetilde{C}(\mathbf{D}(u)+\mathbf{D}(v))
$$

Therefore, recalling Proposition 3.10, we deduce that for every $\gamma \in \mathbb{R}$

$$
\widetilde{\mathcal{E}_{\gamma}}(u, v)<+\infty \quad \forall u, v \in W^{1,2}\left(B^{n}, \mathbb{S}^{2}\right) .
$$

Recalling the notation in (3.5), (3.7), and (4.5), we obtain:

Theorem 6.1. Let $n \geq 2$ and let $\gamma>\max \left\{\gamma_{s}(r), \gamma_{c}(r)\right\}$. Then for every $u, v \in W^{1,2}\left(B^{n}, \mathbb{S}^{2}\right)$ we have

$$
\widetilde{\mathcal{E}_{\gamma}}(u, v)=\mathcal{E}_{\gamma}(u, v)+4 \pi \inf \left\{(1+\gamma)\left(\mathbf{M}\left(L_{T_{1}}\right)+\mathbf{M}\left(L_{T_{2}}\right)\right)+\gamma\left(\Psi\left(L_{T_{1}}\right)+\Psi\left(L_{T_{2}}\right)\right) \mid T_{1} \in \mathcal{T}_{u}, T_{2} \in \mathcal{T}_{v}\right\} .
$$

The "non-local" term $\Psi(L)$ can be more explicitly expressed, using the notation in (5.1), as

$$
\begin{aligned}
\Psi(L) & =\int_{B^{n}} \frac{1}{\left|\widetilde{B}_{r}(x)\right|}\left(\int_{B^{n}} \theta(y) \mathbb{I}_{\widetilde{B}_{r}(x)}(y) \mathrm{d}\left(\mathcal{H}^{n-2}\llcorner\mathcal{L})(y)\right) \mathrm{d} x\right. \\
& =\int_{B^{n}} \theta(y) \mathrm{d}\left(\mathcal{H}^{n-2}\llcorner\mathcal{L})(y) \int_{B^{n}} \frac{1}{\left|\widetilde{B}_{r}(x)\right|} \mathbb{I}_{\widetilde{B}_{r}(y)}(x) \mathrm{d} x\right. \\
& =\int_{\mathcal{L}} \theta(y) k_{r}(y) \mathrm{d} \mathcal{H}^{n-2}(y),
\end{aligned}
$$

where $\theta$ is the multiplicity of the current $L$ and $\mathcal{L}=\{x: \theta(x) \neq 0\}$.

We recall (see Rem. 3.11) that the relaxed Dirichlet energy is computed by considering the "optimal" integral connection of the singular set of $u$. The presence of the term $\Psi(L)$ suggests that for $\gamma \neq 0$, the infimum in (6.2) in general may be strictly lower than the value

$$
(1+\gamma)\left(\mathbf{M}\left(L_{u}\right)+\mathbf{M}\left(L_{v}\right)\right)+\gamma\left(\Psi\left(L_{u}\right)+\Psi\left(L_{v}\right)\right)
$$

where, for $w=u, v$, the current $L_{w} \in \mathcal{R}_{n-2}\left(B^{n}\right)$ is an integral minimal connections of $\mathbb{P}(w)$.

\section{Approximation and density results}

In the proof of the upper bound in Theorem 6.1, we shall make use of an approximation result for the functional

$$
\mathcal{F}_{\gamma}(T)=(1+\gamma) \mathbf{D}(T)+\gamma \Phi(T)
$$

already introduced in (4.11) during the proof of Theorem 4.2. 
Theorem 6.2. Let $\gamma \in \mathbb{R}$. For every $T \in \operatorname{cart}^{2,1}\left(B^{n} \times \mathbb{S}^{2}\right)$, there exists a sequence of smooth maps $\left\{u_{k}\right\} \subset$ $C^{\infty}\left(B^{n}, \mathbb{S}^{2}\right)$ such that $G_{u_{k}} \rightarrow T$ weakly in $\mathcal{D}_{n}\left(B^{n} \times \mathbb{S}^{2}\right)$ as $k \rightarrow \infty$ and

$$
\lim _{k \rightarrow \infty} \mathcal{F}_{\gamma}\left(G_{u_{k}}\right)=\mathcal{F}_{\gamma}(T)
$$

Proof. Write

$$
T=G_{u_{T}}+L_{T} \times \llbracket \mathbb{S}^{2} \rrbracket
$$

as in (3.5), and let $\left\{u_{k}\right\}$ be the smooth approximating sequence given by Theorem 3.9, so that

$$
G_{u_{k}} \rightarrow T \text { and } \lim _{k \rightarrow \infty} \mathbf{D}\left(u_{k}\right)=\mathbf{D}(T) .
$$

Recalling the definition of $\Phi(T)$ in (4.10) we have

$$
\mathcal{F}_{\gamma}\left(G_{u_{k}}\right)=\int_{B^{n}} f_{\gamma}\left(u_{k}, x\right) \mathrm{d} x \quad \text { for every } k,
$$

where

$$
f_{\gamma}\left(u_{k}, x_{0}\right):=\frac{1+\gamma}{2} f_{B^{n}}\left|D u_{k}\right|^{2} \mathrm{~d} x+\frac{\gamma}{2} f_{\widetilde{B}_{r}\left(x_{0}\right)}\left|D u_{k}\right|^{2} \mathrm{~d} x
$$

We now apply Lemma 4.4 with $L$ given by the set of points with positive multiplicity of the current $L_{T} \in$ $\mathcal{R}_{n-2}\left(B^{n}\right)$ in (3.8), obtaining by (6.4) that

$$
\lim _{k \rightarrow \infty} \mathbf{D}\left(u_{k}, \widetilde{B}_{r}\left(x_{0}\right)\right)=\mathbf{D}\left(T, \widetilde{B}_{r}\left(x_{0}\right) \times \mathbb{S}^{2}\right) \quad \text { for } \mathcal{L}^{n} \text {-a.e. } x_{0} \in B^{n} .
$$

Using (4.7), the approximating functions $u_{k}$ also satisfy the estimate

$$
\left|f_{\gamma}\left(u_{k}, x_{0}\right)\right| \leq\left(\frac{|1+\gamma|}{\left|B^{n}\right|}+\frac{|\gamma|}{\left|B^{n}\right|} \frac{1}{c(r) r^{n}}\right) \mathbf{D}\left(u_{k}\right) \quad \forall x_{0} \in B^{n} .
$$

Therefore, recalling (4.12), combining (6.4), (6.5) and (6.6), and applying the dominate convergence theorem, we get

$$
\begin{aligned}
\mathcal{F}_{\gamma}\left(G_{u_{k}}\right) & =\int_{B^{n}}\left(\frac{1+\gamma}{\left|B^{n}\right|} \mathbf{D}\left(u_{k}\right)+\frac{\gamma}{\left|\widetilde{B}_{r}(x)\right|} \mathbf{D}\left(u_{k}, \widetilde{B}_{r}(x)\right)\right) \mathrm{d} x \\
& \rightarrow \int_{B^{n}}\left(\frac{1+\gamma}{\left|B^{n}\right|} \mathbf{D}(T)+\frac{\gamma}{\left|\widetilde{B}_{r}(x)\right|} \mathbf{D}\left(T, \widetilde{B}_{r}(x) \times \mathbb{S}^{2}\right)\right) \mathrm{d} x=\mathcal{F}_{\gamma}(T),
\end{aligned}
$$

which concludes the proof.

We now observe that from (4.9) we have

$$
\mathcal{E}_{\gamma}\left(T_{1}, T_{2}\right)=\mathcal{F}_{\gamma}\left(T_{1}\right)+\mathcal{F}_{\gamma}\left(T_{2}\right)-\gamma\left(C\left(u_{1}, u_{2}\right)+C\left(u_{2}, u_{1}\right)\right) .
$$

Thus, recalling the continuity of the last term proved in the first step of the proof of Theorem 4.2, as an immediate consequence of Theorem 6.2 we obtain:

Corollary 6.3. Let $\gamma \in \mathbb{R}$. Let $T_{1}, T_{2} \in \operatorname{cart}^{2,1}\left(B^{n} \times \mathbb{S}^{2}\right)$, and write

$$
T_{i}=G_{u_{T_{i}}}+L_{T_{i}} \times \llbracket \mathbb{S}^{2} \rrbracket \quad i=1,2 .
$$


Then there exist two sequences of smooth maps $\left\{u_{k}^{i}\right\} \subset C^{\infty}\left(B^{n}, \mathbb{S}^{2}\right), i=1,2$, such that $G_{u_{k}^{i}} \rightarrow T_{i}$ weakly in $\mathcal{D}_{n}\left(B^{n} \times \mathbb{S}^{2}\right)$ as $k \rightarrow \infty$, for $i=1,2$, and

$$
\begin{aligned}
\lim _{k \rightarrow \infty} \mathcal{E}_{\gamma}\left(u_{k}^{1}, u_{k}^{2}\right) & =\mathcal{E}_{\gamma}\left(T_{1}, T_{2}\right) \\
& =\mathcal{E}_{\gamma}\left(u_{T_{1}}, u_{T_{2}}\right)+4 \pi(1+\gamma)\left(\mathbf{M}\left(L_{T_{1}}\right)+\mathbf{M}\left(L_{T_{2}}\right)\right)+4 \pi \gamma\left(\Psi\left(L_{T_{1}}\right)+\Psi\left(L_{T_{2}}\right)\right) .
\end{aligned}
$$

We are now in the position of showing the relaxation formula provided by Theorem 6.1.

Proof of Theorem 6.1. We prove separately the lower bound and the upper bound for $\widetilde{\mathcal{E}_{\gamma}}(u, v)$ in $(6.2)$, for any $u, v \in W^{1,2}\left(B^{n}, \mathbb{S}^{2}\right)$.

Step 1. Lower bound. On account of (6.1), we can find two sequences $\left\{u_{k}\right\},\left\{v_{k}\right\} \subset C^{\infty}\left(B^{n}, \mathbb{S}^{2}\right)$ such that

$$
u_{k} \rightarrow u, v_{k} \rightarrow v \quad \text { weakly in } W^{1,2} \quad \text { and } \quad \sup _{k} \mathcal{E}_{\gamma}\left(u_{k}, v_{k}\right)<+\infty .
$$

By Theorem 5.1 we have that $\sup _{k}\left(\mathbf{D}\left(u_{k}\right)+\mathbf{D}\left(v_{k}\right)\right)<\infty$. Therefore, by Proposition 3.8, possibly passing to a (not relabelled) subsequence, we have that $\lim _{k} \mathcal{E}_{\gamma}\left(u_{k}, v_{k}\right)=\liminf \mathcal{E}_{k} \mathcal{E}_{\gamma}\left(u_{k}, v_{k}\right)$ and $G_{u_{k}} \rightarrow T_{1}$ and $G_{v_{k}} \rightarrow T_{2}$ weakly in $\mathcal{D}_{n}\left(B^{n} \times \mathbb{S}^{2}\right)$ for some Cartesian currents $T_{1}, T_{2} \in \operatorname{cart}^{2,1}\left(B^{n} \times \mathbb{S}^{2}\right)$. We write

$$
T_{i}=G_{u_{T_{i}}}+L_{T_{i}} \times \llbracket \mathbb{S}^{2} \rrbracket \quad i=1,2 .
$$

By the weak convergence (6.7), it turns out that $u_{T_{1}}=u$ and $u_{T_{2}}=v$, thus

$$
\mathcal{E}_{\gamma}\left(u_{T_{1}}, u_{T_{2}}\right)=\mathcal{E}_{\gamma}(u, v) .
$$

Moreover, by Theorem 4.2 we have

$$
\mathcal{E}_{\gamma}\left(T_{1}, T_{2}\right) \leq \liminf _{k \rightarrow \infty} \mathcal{E}_{\gamma}\left(u_{k}, v_{k}\right)
$$

From (6.9) and (4.4) this implies

$$
\mathcal{E}_{\gamma}(u, v)+4 \pi(1+\gamma)\left(\mathbf{M}\left(L_{T_{1}}\right)+\mathbf{M}\left(L_{T_{2}}\right)\right)+4 \pi \gamma\left(\Psi\left(L_{T_{1}}\right)+\Psi\left(L_{T_{2}}\right)\right) \leq \liminf _{k \rightarrow \infty} \mathcal{E}_{\gamma}\left(u_{k}, v_{k}\right) .
$$

The lower bound for $\widetilde{\mathcal{E}_{\gamma}}(u, v)$ in (6.2) follows by taking the infimum on both sides.

Step 2. Upper bound. We consider any couple of Cartesian currents $T_{1}, T_{2} \in \operatorname{cart}^{2,1}\left(B^{n} \times \mathbb{S}^{2}\right)$ such that $T_{1} \in \mathcal{T}_{u}$ and $T_{2} \in \mathcal{T}_{v}$. Taking two sequences $\left\{u_{k}\right\},\left\{v_{k}\right\} \subset C^{\infty}\left(B^{n}, \mathbb{S}^{2}\right)$ as in Corollary 6.3, we deduce

$$
\widetilde{\mathcal{E}_{\gamma}}(u, v) \leq \lim _{k} \mathcal{E}_{\gamma}\left(u_{k}, v_{k}\right)=\mathcal{E}_{\gamma}(u, v)+4 \pi(1+\gamma)\left(\mathbf{M}\left(L_{T_{1}}\right)+\mathbf{M}\left(L_{T_{2}}\right)\right)+4 \pi \gamma\left(\Psi\left(L_{T_{1}}\right)+\Psi\left(L_{T_{2}}\right)\right) .
$$

The upper bound for $\widetilde{\mathcal{E}_{\gamma}}(u, v)$ in (6.2) follows by taking the infimum on the right hand side.

\section{Positivity of the relaxed functional}

From Theorem 5.1 and Proposition 3.10 we immediately deduce the following result.

Corollary 6.4. Let $\gamma>\gamma_{c}(r)$. Then for every $u, v \in W^{1,2}\left(B^{n}, \mathbb{S}^{2}\right)$ we have

$$
\widetilde{\mathcal{E}_{\gamma}}(u, v) \geq C(\widetilde{\mathbf{D}}(u)+\widetilde{\mathbf{D}}(v)),
$$

where $C=C(\gamma, r)>0$ is given by Theorem 5.1.

Notice that this result is significant for the values of $\gamma$ such that the energy $\mathcal{E}_{\gamma}$ is coercive but not lower semicontinuous: indeed, in these cases the explicit expression of the relaxed functional given in Theorem 6.1 is missing. This happens e.g. for every $r>0$ very small. In fact, from the analysis of the behavior of the thresholds $\gamma_{s}(r)$ and $\gamma_{c}(r)$ we can find a radius $r_{0} \in(0,2)$, depending on $n$, for which

$$
\gamma_{c}(r)<\gamma_{s}(r) \text { for every } r \text { such that } 0<r<r_{0} .
$$




\section{AdDitionAl REMARKS AND LACK OF OPTIMALITY OF THE THRESHOLDS}

In this section we first specialize the previous results to the two-dimensional case. Moreover, we construct explicit examples illustrating that the energy $\mathcal{E}_{\gamma}$ may be not lower semicontinuous or not coercive for suitably chosen values of $\gamma$. We also comment on the lack of optimality of the thresholds $\gamma_{s}(r)$ and $\gamma_{c}(r)$.

\section{The two-dimensional case}

Assume $n=2$, and let $T \in \operatorname{cart}^{2,1}\left(B^{2} \times \mathbb{S}^{2}\right)$, so that by (3.5) we have

$$
T=G_{u_{T}}+L_{T} \times \llbracket \mathbb{S}^{2} \rrbracket, \quad u_{T} \in W^{1,2}\left(B^{2}, \mathbb{S}^{2}\right), \quad L_{T}=\sum_{i=1}^{m} \sigma_{i} \delta_{a_{i}}
$$

for some $m \in \mathbb{N}, \sigma_{i} \in \mathbb{Z}$, and $a_{i} \in B^{2}$, where $\delta_{a}$ is the unit Dirac mass at the point $a$. We may assume that each $\sigma_{i} \neq 0$ and that the points $a_{i}$ are distinct. We thus have

$$
\mathbf{D}(T)=\mathbf{D}\left(u_{T}\right)+4 \pi \mathbf{M}\left(L_{T}\right), \quad \mathbf{M}\left(L_{T}\right)=\sum_{i=1}^{m}\left|\sigma_{i}\right|
$$

and for every Borel set $B \subset B^{2}$

$$
\mathbf{D}\left(T, B \times \mathbb{S}^{2}\right)=\mathbf{D}\left(u_{T}, B\right)+4 \pi \mathbf{M}\left(L_{T}\llcorner B), \quad \mathbf{M}\left(L_{T}\llcorner B)=\sum_{i=1}^{m}\left|\sigma_{i}\right| \delta_{a_{i}}(B) .\right.\right.
$$

As a consequence, for the functional (4.5) we compute

$$
\begin{aligned}
\Psi\left(L_{T}\right) & =\int_{B^{2}} \frac{1}{\left|\widetilde{B}_{r}(x)\right|} \sum_{i=1}^{m}\left|\sigma_{i}\right| \delta_{a_{i}}\left(\widetilde{B}_{r}(x)\right) \mathrm{d} x \\
& =\sum_{i=1}^{m}\left|\sigma_{i}\right| \int_{B^{2}} \frac{1}{\left|\widetilde{B}_{r}(x)\right|} \delta_{x}\left(\widetilde{B}_{r}\left(a_{i}\right)\right) \mathrm{d} x=\sum_{i=1}^{m}\left|\sigma_{i}\right| k_{r}\left(a_{i}\right),
\end{aligned}
$$

where $k_{r}(y)$ has been defined in (5.1). According to (6.2), this yields that

$$
(1+\gamma) \mathbf{M}\left(L_{T}\right)+\gamma \Psi\left(L_{T}\right)=\sum_{i=1}^{m}\left|\sigma_{i}\right|\left(1+\gamma\left(1+k_{r}\left(a_{i}\right)\right)\right) .
$$

In particular, for $\gamma<0$ we have by $(5.2)$

$$
(1+\gamma) \mathbf{M}\left(L_{T}\right)+\gamma \Psi\left(L_{T}\right) \geq(1+\gamma(1+K(r))) \sum_{i=1}^{m}\left|\sigma_{i}\right| .
$$

Therefore,

$$
\gamma \geq-\frac{1}{1+K(r)} \quad \Longrightarrow \quad(1+\gamma) \mathbf{M}\left(L_{T}\right)+\gamma \Psi\left(L_{T}\right) \geq 0 \quad \forall T \in \operatorname{cart}^{2,1}\left(B^{2} \times \mathbb{S}^{2}\right) .
$$

On the other hand, recalling that there exists a radius $\rho=\rho(r, n)$ between $1-r$ and 1 such that the supremum $K(r)$ in (5.2) is attained for any $y$ with $\|y\|=\rho$, and taking a current with just one singular point $a_{1}$ chosen so that $k_{r}\left(a_{1}\right)=K(r)$, we get the converse arrow, thus

$$
\gamma \geq-\frac{1}{1+K(r)} \quad \Longleftrightarrow \quad(1+\gamma) \mathbf{M}\left(L_{T}\right)+\gamma \Psi\left(L_{T}\right) \geq 0 \quad \forall T \in \operatorname{cart}^{2,1}\left(B^{2} \times \mathbb{S}^{2}\right)
$$


Since our coercivity threshold $\gamma_{c}(r)$ satisfies

$$
\gamma_{c}(r)>-\frac{1}{1+K(r)}
$$

using Schoen-Uhlenbeck density theorem [11] and Remark 3.11 we deduce that Theorem 6.1 and Corollary 6.4 reduce as follows.

Proposition 7.1. Let $n=2$. If $\gamma>\max \left\{\gamma_{s}(r), \gamma_{c}(r)\right\}$, then for every $(u, v) \in W^{1,2}\left(B^{2} \times \mathbb{S}^{2}\right)$ we have

$$
\widetilde{\mathcal{E}_{\gamma}}(u, v)=\mathcal{E}_{\gamma}(u, v) .
$$

If we just assume $\gamma>\gamma_{c}(r)$, then for every $(u, v) \in W^{1,2}\left(B^{2} \times \mathbb{S}^{2}\right)$ we have

$$
\widetilde{\mathcal{E}_{\gamma}}(u, v) \geq C(\mathbf{D}(u)+\mathbf{D}(v)),
$$

where $C=C(\gamma, r)>0$ is given by Theorem 5.1.

\section{An example about the lower semicontinuity}

We give an example in dimension $n=2$ showing that the lower semicontinuity property of Theorem 4.2 fails to hold if

$$
\gamma<-\frac{1}{1+K(r)}
$$

To this purpose, let $T \in \operatorname{cart}^{2,1}\left(B^{2} \times \mathbb{S}^{2}\right)$ as in (7.1), where the function $u_{T} \equiv P \in \mathbb{S}^{2}$ is constant and the points $a_{i}$ are distinct. Since $\mathbf{D}\left(u_{T}\right)=0$, the functional $\Phi(T)$ defined in (4.10) reduces to

$$
\Phi(T)=4 \pi \Psi\left(L_{T}\right)
$$

Recalling (4.11) we thus get

$$
\mathcal{F}_{\gamma}(T)=4 \pi\left((1+\gamma) \mathbf{M}\left(L_{T}\right)+\gamma \Psi\left(L_{T}\right)\right)
$$

so that by $(7.2)$ we have

$$
\mathcal{F}_{\gamma}(T)=4 \pi \sum_{i=1}^{m}\left|\sigma_{i}\right|\left(1+\gamma\left(1+k_{r}\left(a_{i}\right)\right)\right) .
$$

Assume now that $\sigma_{i}= \pm 1$. By (7.3) we have

$$
\mathcal{F}_{\gamma}(T)=4 \pi m(1+\gamma(1+f(T, r))
$$

where the average $f(T, r)$ is a positive number which depends on the position of the points $a_{i}$ in $B^{2}$, and by (5.4) lies between $c(r)$ and $1 / c(r)$; in particular it also satisfies

$$
f(T, r) \leq K(r)
$$

Now, for $r$ small we have $f(T, r)=1$ if all the points $a_{i}$ are not too close to the boundary, namely if $\left|a_{i}\right|<1-2 r$. On the other hand, choosing the points $a_{i} \in B^{2}$ in such a way that $\left\|a_{i}\right\|=\rho(r, 2)$, we have $k_{r}\left(a_{i}\right)=K(r)$ and hence

$$
\mathcal{F}_{\gamma}(T)=4 \pi m(1+\gamma(1+K(r)))
$$

Set

$$
T_{1}=T_{2}=T_{h}^{2}=G_{P}
$$

the graph of the constant $P \in \mathbb{S}^{2}$, and

$$
T_{h}^{1}:=G_{P}+\left(\delta_{a_{h}^{+}}-\delta_{a_{h}^{-}}\right) \times \llbracket \mathbb{S}^{2} \rrbracket
$$


where the points $a_{h}^{ \pm}$are distinct, have the same distance from the boundary and converge to the origin, so that the singularities cancel out and $T_{h}^{1} \rightarrow G_{P}=T_{1}$. A counterexample to lower semicontinuity will be obtained if we show that

$$
\mathcal{F}_{\gamma}\left(T_{1}\right)>\liminf _{h \rightarrow \infty} \mathcal{F}_{\gamma}\left(T_{h}^{1}\right) .
$$

But $\mathcal{F}_{\gamma}\left(T_{1}\right)=0$, whereas for $h$ sufficiently large we have $\left\|a_{h}^{ \pm}\right\|<1-2 r$, thus $k_{r}\left(a_{h}^{ \pm}\right)=1$ and

$$
\mathcal{F}_{\gamma}\left(T_{h}^{1}\right)=8 \pi(1+2 \gamma)<0=\mathcal{F}_{\gamma}\left(T_{1}\right)
$$

as soon as $\gamma<-1 / 2$. To get a sharper estimate, it is enough to change the choice of the singular points: the couple $a_{h}^{ \pm}$should satisfy $\left\|a_{h}^{ \pm}\right\|=\rho(r, 2)$ and converge to a point, so that

$$
\mathcal{F}_{\gamma}\left(T_{h}^{1}\right)=8 \pi(1+\gamma(1+K(r)))<0=\mathcal{F}_{\gamma}\left(T_{1}\right)
$$

as soon as

$$
\gamma<-\frac{1}{1+K(r)}
$$

Since the semicontinuity threshold $\gamma_{s}(r)$ in (4.6) satisfies $\gamma_{s}(r) \rightarrow 0^{-}$as $r \rightarrow 0^{+}$, this suggests that $\gamma_{s}(r)$ should be far from being optimal, for $r$ small.

\section{Coercivity and radius of interaction}

The estimate in Theorem 5.1 is quite rough: indeed the absolute constant of (5.2) does not distinguish between "good" balls $B$, well within $B^{n}$, and balls close to the boundary. If the support of $f$ is far (i.e. at a distance greater than $2 r$ ) from $\partial B^{n}$ then (5.3) trivially becomes

$$
\int_{B^{n}} f_{\widetilde{B}_{r}(x)} f(y) \mathrm{d} y \mathrm{~d} x=\int_{B^{n}} f(x) \mathrm{d} x
$$

thus $K(r)$ is only needed at points near the boundary. On the other hand, due to the nonlocality of the problem a global constant seems to be the main way to control the double integrals with a single integral. Indeed in (5.5) one discards, without getting any coercivity benefit, the averages of $\left|\eta D u(x)+\eta^{-1} D v(y)\right|^{2}$ and of $\left|\eta D v(x)+\eta^{-1} D u(y)\right|^{2}$, and it is impossible that both vanish everywhere.

We now construct an example with loss of coercivity for $\gamma$ satisfying (7.5), which is (strictly) less than our coercivity threshold $\gamma_{c}(r)$.

Let $n=2$ and let $u_{P} \equiv P$ and $v_{Q} \equiv Q$ be constant maps, $P, Q \in \mathbb{S}^{2}$. We claim that for every positive integer $m \in \mathbb{N}^{+}$we can find two sequences $\left\{u_{k}\right\},\left\{v_{k}\right\}$ of smooth functions from $B^{2}$ into $\mathbb{S}^{2}$ such that $u_{k} \rightarrow u_{P}$ and $v_{k} \rightarrow v_{Q}$ weakly in $W^{1,2}$, with $\sup _{k}\left(\mathbf{D}\left(u_{k}\right)+\mathbf{D}\left(u_{k}\right)\right)<\infty$, and

$$
\mathcal{E}_{\gamma}\left(u_{k}, v_{k}\right) \leq 8 \pi m(1+\gamma(1+K(r)))+\frac{1}{m} \quad \forall k .
$$

Therefore, if $(1+\gamma(1+K(r)))<0$, i.e., if (7.5) holds, the claim gives that the relaxed functional (1.5) satisfies

$$
\widetilde{\mathcal{E}_{\gamma}}\left(u_{P}, v_{Q}\right)=-\infty
$$

To prove the claim, we set

$$
T_{h}:=G_{P}+m\left(\delta_{a_{h}^{+}}-\delta_{a_{h}^{-}}\right) \times \llbracket \mathbb{S}^{2} \rrbracket
$$

where we choose in an optimal position the points of mass concentration, in such a way that $k_{r}\left(a_{h}^{ \pm}\right)=K(r)$. Therefore, compare (4.11), if $a_{h}^{+} \neq a_{h}^{-}$, arguing as above we find that

$$
\mathcal{F}_{\gamma}\left(T_{h}\right)=8 \pi m(1+\gamma(1+K(r)))
$$


Moreover, we may choose the points $a_{h}^{ \pm}$so that $\left|a_{h}^{+}-a_{h}^{-}\right| \rightarrow 0$ as $h \rightarrow \infty$. This clearly yields that $T_{h} \rightarrow G_{P}$ weakly in $\mathcal{D}_{2}\left(B^{2} \times \mathbb{S}^{2}\right)$, as a cancellation occurs when passing to the limit. By Theorem 6.2 , for every $h$ we find a sequence of smooth maps $\left\{u_{k}^{h}\right\} \subset C^{\infty}\left(B^{2}, \mathbb{S}^{2}\right)$ such that $G_{u_{k}^{h}} \rightarrow T_{h}$ weakly in $\mathcal{D}_{2}\left(B^{2} \times \mathbb{S}^{2}\right)$ and $\mathcal{F}_{\gamma}\left(G_{u_{k}^{h}}\right) \rightarrow \mathcal{F}_{\gamma}\left(T_{h}\right)$ as $k \rightarrow \infty$. Setting $v_{k}^{h} \equiv Q$, and arguing as for the proof of Corollary 6.3, a diagonal argument yields the claim.

\section{ENERGy OF MULTifunCtions}

In this section we describe an approach to our problem within the setting of multifunctions.

\section{Multifunctions}

We first introduce an equivalence relation on couples of Sobolev functions.

Definition 8.1. For any given couples $(u, v)$ and $(\widetilde{u}, \widetilde{v})$ of functions in $W^{1,2}\left(B^{n}, \mathbb{S}^{2}\right)$, we say that $(u, v) \sim(\widetilde{u}, \widetilde{v})$ if

$$
\mathcal{L}^{n}\left(\left\{x \in B^{n} \mid\{u(x), v(x)\} \neq\{\widetilde{u}(x), \widetilde{v}(x)\}\right\}\right)=0 .
$$

This equivalence relation can be written in terms of currents carried by the graphs as

$$
(u, v) \sim(\widetilde{u}, \widetilde{v}) \Longleftrightarrow\left(G_{u}+G_{v}\right)(\omega)=\left(G_{\widetilde{u}}+G_{\widetilde{v}}\right)(\omega) \quad \forall \omega \in \mathcal{D}^{n}\left(B^{n} \times \mathbb{S}^{2}\right)
$$

The characterization in (8.1) follows from the equality in (3.1), splitting the integral on the sets

$$
A:=\left\{x \in B^{n} \mid u(x)=\widetilde{u}(x)\right\}, \quad B:=B^{n} \backslash A
$$

and noticing that (from the locality of the gradient operator in the space of Sobolev maps) we have $D u=D \widetilde{u}$ and $D v=D \widetilde{v} \mathcal{L}^{n}$-a.e. on $A$, whereas we have $D u=D \widetilde{v}$ and $D v=D \widetilde{u} \mathcal{L}^{n}$-a.e. on $B$. In particular, if $(u, v) \sim(\widetilde{u}, \widetilde{v})$ we have that

$$
\int_{B^{n}}(\varphi(x, u(x))+\varphi(x, v(x))) \mathrm{d} x=\int_{B^{n}}(\varphi(x, \widetilde{u}(x))+\varphi(x, \widetilde{v}(x))) \mathrm{d} x
$$

for every test function $\varphi=\varphi(x, y) \in C_{c}^{\infty}\left(B^{n} \times \mathbb{R}^{3}\right)$.

Definition 8.2. We denote by $M_{2} W^{1,2}\left(B^{n}, \mathbb{S}^{2}\right)$ the set of equivalence classes $[u, v]$ of couples $(u, v)$ of functions in $W^{1,2}\left(B^{n}, \mathbb{S}^{2}\right)$ under the equivalence relation given by Definition 8.1.

We are led by (8.2) to consider the following weak convergence:

Definition 8.3. We say that $\left[u_{k}, v_{k}\right] \rightarrow[u, v]$ weakly in $M_{2} W^{1,2}$ if

$$
\lim _{k \rightarrow \infty} \int_{B^{n}}\left(\varphi\left(x, u_{k}(x)\right)+\varphi\left(x, v_{k}(x)\right)\right) \mathrm{d} x=\int_{B^{n}}(\varphi(x, u(x))+\varphi(x, v(x))) \mathrm{d} x
$$

for every $\varphi \in C_{c}^{\infty}\left(B^{n} \times \mathbb{R}^{3}\right)$.

In particular, if $u_{k} \rightarrow u$ and $v_{k} \rightarrow v$ strongly in $L_{\text {loc }}^{1}\left(B^{n}\right)$, we have $\left[u_{k}, v_{k}\right] \rightarrow[u, v]$ weakly in $M_{2} W^{1,2}$. We also explicitly notice that if $u_{k} \rightarrow u$ and $v_{k} \rightarrow v$ weakly in $W^{1,2}\left(B^{n}, \mathbb{R}^{3}\right)$, then $\left[u_{k}, v_{k}\right] \rightarrow[u, v]$ weakly in $M_{2} W^{1,2}$.

In terms of graphs, the convergence just introduced corresponds to the weak convergence

$$
\lim _{k \rightarrow \infty}\left(G_{u_{k}}+G_{v_{k}}\right)(\varphi(x, y) \mathrm{d} x)=\left(G_{u}+G_{v}\right)(\varphi(x, y) \mathrm{d} x) \quad \forall \varphi \in C_{c}^{\infty}\left(B^{n} \times \mathbb{R}^{3}\right),
$$

i.e., to the dual convergence on currents restricted to horizontal test $n$-forms $\varphi(x, y) \mathrm{d} x$. 


\section{Energy of multifunctions}

As will be shown by the example in Section 9, the energy $\mathcal{E}_{\gamma}(u, v)$ may depend on the choice of the representative in the equivalence class $[u, v]$. We therefore define the energy $\mathcal{E}_{\gamma}[u, v]$ of an element $[u, v]$ in $M_{2} W^{1,2}\left(B^{n}, \mathbb{S}^{2}\right)$ through an additional minimization procedure, that is

$$
E_{\gamma}[u, v]:=\inf \left\{\mathcal{E}_{\gamma}(\widetilde{u}, \widetilde{v}) \mid(\widetilde{u}, \widetilde{v}) \sim(u, v)\right\} .
$$

One may then consider the following relaxed energy on the class $M_{2} W^{1,2}\left(B^{n}, \mathbb{S}^{2}\right)$

$$
\bar{E}_{\gamma}[u, v]:=\inf \left\{\liminf _{k \rightarrow \infty} E_{\gamma}\left[u_{k}, v_{k}\right] \quad \mid \quad\left\{u_{k}\right\},\left\{v_{k}\right\} \subset C^{\infty}\left(B^{n}, \mathbb{S}^{2}\right), \quad\left[u_{k}, v_{k}\right] \rightarrow[u, v] \text { weakly in } M_{2} W^{1,2}\right\} .
$$

More precisely, $\bar{E}_{\gamma}[u, v]$ is the lower semicontinuous envelope of the non-negative functional defined on $M_{2} W^{1,2}\left(B^{n}, \mathbb{S}^{2}\right)$ by

$$
[\widetilde{u}, \widetilde{v}] \mapsto \begin{cases}E_{\gamma}[\widetilde{u}, \widetilde{v}] & \text { if }[\widetilde{u}, \widetilde{v}] \text { is smooth } \\ +\infty & \text { otherwise }\end{cases}
$$

where $[\widetilde{u}, \widetilde{v}]$ is said to be smooth if there exists a representative $(u, v) \in[\widetilde{u}, \widetilde{v}]$ such that $u, v \in C^{\infty}\left(B^{n}, \mathbb{S}^{2}\right)$.

However, we do not know whether for $u, v \in C^{\infty}\left(B^{n}, \mathbb{S}^{2}\right)$ we have $E_{\gamma}[u, v]=\mathcal{E}_{\gamma}(u, v)$, as in principle it may happen that there exists a couple of non-smooth $W^{1,2}$-functions $(\widetilde{u}, \widetilde{v}) \in[u, v]$ such that $\mathcal{E}_{\gamma}(\widetilde{u}, \widetilde{v})<\mathcal{E}_{\gamma}(u, v)$. For this reason, it is difficult to find an explicit formula for (8.5). We argue in a different way, that is suggested by a relaxation result for equivalence classes of currents.

\section{Equivalence classes of currents}

We first introduce an equivalence relation on couples of Cartesian currents.

Definition 8.4. For any given couples $(S, T)$ and $(\widetilde{S}, \widetilde{T})$ of currents in $\operatorname{cart}^{2,1}\left(B^{n} \times \mathbb{S}^{2}\right)$, we say that $(S, T) \simeq$ $(\widetilde{S}, \widetilde{T})$ if

$$
(S+T)(\omega)=(\widetilde{S}+\widetilde{T})(\omega) \quad \forall \omega \in \mathcal{D}^{n}\left(B^{n} \times \mathbb{S}^{2}\right)
$$

We also denote by $M_{2} \operatorname{cart}^{2,1}\left(B^{n} \times \mathbb{S}^{2}\right)$ the corresponding set of equivalence classes $[S, T]$. Moreover, we say that $\left[S_{k}, T_{k}\right] \rightarrow[S, T]$ weakly in $M_{2}$ cart $^{2,1}$ if

$$
\left(S_{k}+T_{k}\right)(\omega) \rightarrow(S+T)(\omega) \quad \forall \omega \in \mathcal{D}^{n}\left(B^{n} \times \mathbb{S}^{2}\right) .
$$

Notice that if we denote by $u_{T} \in W^{1,2}\left(B^{n}, \mathbb{S}^{2}\right)$ the Sobolev function corresponding to a current $T \in$ $\operatorname{cart}^{2,1}\left(B^{n} \times \mathbb{S}^{2}\right)$, then we have:

(i) $(S, T) \simeq(\widetilde{S}, \widetilde{T}) \Rightarrow\left(u_{S}, u_{T}\right) \sim\left(u_{\widetilde{S}}, u_{\widetilde{T}}\right)$;

(ii) $\left[S_{k}, T_{k}\right] \rightarrow[S, T]$ in $M_{2} \operatorname{cart}^{2,1} \stackrel{S}{\Rightarrow}\left[u_{S_{k}}, u_{T_{k}}\right] \rightarrow\left[u_{S}, u_{T}\right]$ in $M_{2} W^{1,2}$.

We then define the energy of an element $[S, T] \in M_{2} \operatorname{cart}^{2,1}\left(B^{n} \times \mathbb{S}^{2}\right)$ as

$$
E_{\gamma}[S, T]:=\inf \left\{\mathcal{E}_{\gamma}(\widetilde{S}, \widetilde{T}) \mid(\widetilde{S}, \widetilde{T}) \simeq(S, T)\right\} .
$$

It turns out that the infimum in the above definition is attained:

Proposition 8.5. If $\gamma>\max \left\{\gamma_{s}(r), \gamma_{c}(r)\right\}$, for every $[S, T] \in M_{2} \operatorname{cart}^{2,1}\left(B^{n} \times \mathbb{S}^{2}\right)$ we have

$$
E_{\gamma}[S, T]=\min \left\{\mathcal{E}_{\gamma}(\widetilde{S}, \widetilde{T}) \mid(\widetilde{S}, \widetilde{T}) \simeq(S, T)\right\} .
$$


Proof. Clearly $E_{\gamma}[S, T]<\infty$. Let $\left\{\left(S_{k}, T_{k}\right)\right\}$ be a minimizing sequence with $\left(S_{k}, T_{k}\right) \simeq(S, T)$. By coercivity we have $\sup _{k}\left(\mathbf{D}\left(S_{k}\right)+\mathbf{D}\left(T_{k}\right)\right)<\infty$. Therefore, by closure-compactness, possibly passing to a subsequence we deduce that $S_{k} \rightarrow \bar{S}$ and $T_{k} \rightarrow \bar{T}$ in $\mathcal{D}_{n}\left(B^{n} \times \mathbb{S}^{2}\right)$ to some $\bar{S}, \bar{T} \in \operatorname{cart}^{2,1}\left(B^{n} \times \mathbb{S}^{2}\right)$, with $(\bar{S}, \bar{T}) \simeq(S, T)$. By lower semicontinuity, we thus have

$$
\mathcal{E}_{\gamma}(\bar{S}, \bar{T}) \leq \liminf _{k \rightarrow \infty} \mathcal{E}_{\gamma}\left(S_{k}, T_{k}\right)=E_{\gamma}[S, T]
$$

that proves the claim.

The corresponding relaxed energy on the class $M_{2} \operatorname{cart}^{2,1}\left(B^{n} \times \mathbb{S}^{2}\right)$ is then defined by

$$
\widetilde{E_{\gamma}}[S, T]:=\inf \left\{\liminf _{k \rightarrow \infty} E_{\gamma}\left[G_{u_{k}}, G_{v_{k}}\right] \mid\left\{u_{k}\right\},\left\{v_{k}\right\} \subset C^{\infty}\left(B^{n}, \mathbb{S}^{2}\right), \quad\left[G_{u_{k}}, G_{v_{k}}\right] \rightarrow[S, T] \text { weakly in } M_{2} \text { cart }^{2,1}\right\} .
$$

The following equality holds:

Proposition 8.6. If $\gamma>\max \left\{\gamma_{s}(r), \gamma_{c}(r)\right\}$, for every $[S, T] \in M_{2} \operatorname{cart}^{2,1}\left(B^{n} \times \mathbb{S}^{2}\right)$ we have

$$
\widetilde{E_{\gamma}}[S, T]=E_{\gamma}[S, T] .
$$

Proof. We prove separately the lower and the upper bound.

Step 1. Lower bound. We can assume without loss of generality that $\widetilde{E_{\gamma}}[S, T]<\infty$, otherwise the lower bound is trivial. Let $\left\{\left(u_{k}, v_{k}\right)\right\}$ be a sequence of couples of smooth functions in $C^{\infty}\left(B^{n}, \mathbb{S}^{2}\right)$ such that $\left[G_{u_{k}}, G_{v_{k}}\right] \rightarrow[S, T]$ in $M_{2}$ cart $^{2,1}$, with $\sup _{k} E_{\gamma}\left[G_{u_{k}}, G_{v_{k}}\right]<\infty$. By Proposition 8.5, we have $E_{\gamma}\left[G_{u_{k}}, G_{v_{k}}\right]=\mathcal{E}_{\gamma}\left(S_{k}, T_{k}\right)$ for some $\left(S_{k}, T_{k}\right) \in\left[G_{u_{k}}, G_{v_{k}}\right]$. As in the proof of Proposition 8.5, possibly passing to a subsequence $S_{k} \rightarrow \bar{S}$ and $T_{k} \rightarrow \bar{T}$ in $\mathcal{D}_{n}\left(B^{n} \times \mathbb{S}^{2}\right)$ to some $\bar{S}, \bar{T} \in \operatorname{cart}^{2,1}\left(B^{n} \times \mathbb{S}^{2}\right)$. By lower semicontinuity, we thus have

$$
E_{\gamma}[\bar{S}, \bar{T}] \leq \mathcal{E}_{\gamma}(\bar{S}, \bar{T}) \leq \liminf _{k \rightarrow \infty} \mathcal{E}_{\gamma}\left(S_{k}, T_{k}\right)=\liminf _{k \rightarrow \infty} E_{\gamma}\left[G_{u_{k}}, G_{v_{k}}\right]
$$

Since $\left(G_{u_{k}}+G_{v_{k}}\right)=\left(S_{k}+T_{k}\right) \rightarrow(\bar{S}+\bar{T})$ weakly in $\mathcal{D}_{n}\left(B^{n} \times \mathbb{S}^{2}\right)$, we deduce that $(\bar{S}, \bar{T}) \in[S, T]$ and hence that $E_{\gamma}[S, T]=E_{\gamma}[\bar{S}, \bar{T}]$. This, together with inequality (8.9), gives the lower bound for the relaxed energy $\widetilde{E_{\gamma}}[S, T] \geq E_{\gamma}[S, T]$.

Step 2. Upper bound. By Proposition 8.5, we can find $(\widetilde{S}, \widetilde{T}) \simeq(S, T)$ such that $E_{\gamma}[S, T]=\mathcal{E}_{\gamma}(\widetilde{S}, \widetilde{T})$. By Corollary 6.3 , we let $u_{k}, v_{k} \in C^{\infty}\left(B^{n}, \mathbb{S}^{2}\right)$ such that $\left[G_{u_{k}}, G_{v_{k}}\right] \rightarrow[\widetilde{S}, \widetilde{T}]$ in $M_{2} \operatorname{cart}^{2,1}$ and $\mathcal{E}_{\gamma}\left(G_{u_{k}}, G_{v_{k}}\right) \rightarrow$ $\mathcal{E}_{\gamma}(\widetilde{S}, \widetilde{T})$. Again by Proposition 8.5 we have $E_{\gamma}\left[G_{u_{k}}, G_{v_{k}}\right]=\mathcal{E}_{\gamma}\left(\widetilde{S}_{k}, \widetilde{T}_{k}\right)$ for some $\left(\widetilde{S}_{k}, \widetilde{T}_{k}\right) \in\left[G_{u_{k}}, G_{v_{k}}\right]$, and possibly passing to a subsequence $\widetilde{S}_{k} \rightarrow \bar{S}$ and $\widetilde{T}_{k} \rightarrow \bar{T}$ in $\mathcal{D}_{n}\left(B^{n} \times \mathbb{S}^{2}\right)$ to some $\bar{S}, \bar{T} \in \operatorname{cart}^{2,1}\left(B^{n} \times \mathbb{S}^{2}\right)$. By lower semicontinuity this yields

$$
\mathcal{E}_{\gamma}(\bar{S}, \bar{T}) \leq \liminf _{k \rightarrow \infty} \mathcal{E}_{\gamma}\left(\widetilde{S}_{k}, \widetilde{T}_{k}\right) \leq \lim _{k \rightarrow \infty} \mathcal{E}_{\gamma}\left(G_{u_{k}}, G_{v_{k}}\right)=\mathcal{E}_{\gamma}(\widetilde{S}, \widetilde{T})
$$

Since $\left(\widetilde{S}_{k}, \widetilde{T}_{k}\right) \simeq\left(G_{u_{k}}, G_{v_{k}}\right)$ we have $(\bar{S}, \bar{T}) \simeq(\widetilde{S}, \widetilde{T}) \simeq(S, T)$. This implies, recalling the choice of $(\widetilde{S}, \widetilde{T})$, that

$$
\mathcal{E}_{\gamma}(\widetilde{S}, \widetilde{T})=E_{\gamma}[S, T] \leq \mathcal{E}_{\gamma}(\bar{S}, \bar{T}) .
$$

Combining (8.10) and (8.11) we get

$$
\liminf _{k \rightarrow \infty} E_{\gamma}\left[G_{u_{k}}, G_{v_{k}}\right]=\liminf _{k \rightarrow \infty} \mathcal{E}_{\gamma}\left(\widetilde{S}_{k}, \widetilde{T}_{k}\right)=\lim _{k \rightarrow \infty} \mathcal{E}_{\gamma}\left(G_{u_{k}}, G_{v_{k}}\right)=E_{\gamma}[S, T]
$$

where the first equality is due to the choice of $\left(\widetilde{S}_{k}, \widetilde{T}_{k}\right)$. Up to an extraction of a subsequence this gives $E_{\gamma}\left[G_{u_{k}}, G_{v_{k}}\right] \rightarrow E_{\gamma}[S, T]$, and by the fact that $\left[G_{u_{k}}, G_{v_{k}}\right] \rightarrow[S, T]$ we obtain the upper bound for the relaxed energy $\widetilde{E_{\gamma}}[S, T] \leq E_{\gamma}[S, T]$. 


\section{Relaxed energy of multifunctions}

Motivated by Proposition 8.6, we are led to introduce on the class $M_{2} W^{1,2}\left(B^{n}, \mathbb{S}^{2}\right)$ the relaxed energy

$$
\widetilde{E_{\gamma}}[u, v]:=\inf \left\{\liminf _{k \rightarrow \infty} E_{\gamma}\left[G_{u_{k}}, G_{v_{k}}\right] \quad \mid \quad\left\{u_{k}\right\},\left\{v_{k}\right\} \subset C^{\infty}\left(B^{n}, \mathbb{S}^{2}\right), \quad\left[u_{k}, v_{k}\right] \rightarrow[u, v] \text { weakly in } M_{2} W^{1,2}\right\},
$$

i.e., the lower semicontinuous envelope of the non-negative functional on $M_{2} W^{1,2}\left(B^{n}, \mathbb{S}^{2}\right)$

$$
[\widetilde{u}, \widetilde{v}] \mapsto \begin{cases}\widehat{E_{\gamma}}[\widetilde{u}, \widetilde{v}] & \text { if }[\widetilde{u}, \widetilde{v}] \text { is smooth } \\ +\infty & \text { otherwise }\end{cases}
$$

where $[\widetilde{u}, \widetilde{v}]$ is smooth in the sense of $(8.6)$ and for any such smooth class $[\widetilde{u}, \widetilde{v}]$ we have set

$$
\widehat{E_{\gamma}}[\widetilde{u}, \widetilde{v}]:=E_{\gamma}\left[G_{u}, G_{v}\right] \text { for some } u, v \in C^{\infty}\left(B^{n}, \mathbb{S}^{2}\right) \text { such that }(u, v) \sim(\widetilde{u}, \widetilde{v}) .
$$

The following formula for the relaxed energy (8.12) holds:

Theorem 8.7. Let $n \geq 2$ and let $\gamma>\max \left\{\gamma_{s}(r), \gamma_{c}(r)\right\}$. Then the relaxed energy (8.12) is always finite and for every $[u, v] \in M_{2} W^{1,2}\left(B^{n}, \mathbb{S}^{2}\right)$ we have

$$
\widetilde{E_{\gamma}}[u, v]=\inf \left\{E_{\gamma}[S, T] \mid S \in \mathcal{T}_{\widetilde{u}}, T \in \mathcal{T}_{\widetilde{v}}, \widetilde{u}, \widetilde{v} \in W^{1,2}\left(B^{n}, \mathbb{S}^{2}\right),(\widetilde{u}, \widetilde{v}) \sim(u, v)\right\} .
$$

As a consequence, using property i) after Definition 8.4, by the definitions (4.4) and (8.7) we immediately obtain the following explicit formula for the relaxed energy of multifunctions.

Corollary 8.8. For every $[u, v] \in M_{2} W^{1,2}\left(B^{n}, \mathbb{S}^{2}\right)$ we have

$$
\begin{aligned}
& \widetilde{E_{\gamma}}[u, v]=\inf \left\{\mathcal{E}_{\gamma}(\widetilde{u}, \widetilde{v})+4\right.\left((1+\gamma)\left(\mathbf{M}\left(L_{S}\right)+\mathbf{M}\left(L_{T}\right)\right)+\gamma\left(\Psi\left(L_{S}\right)+\Psi\left(L_{T}\right)\right)\right. \\
&\left.\mid S \in \mathcal{T}_{\widetilde{u}}, T \in \mathcal{T}_{\widetilde{v}}, \widetilde{u}, \widetilde{v} \in W^{1,2}\left(B^{n}, \mathbb{S}^{2}\right),(\widetilde{u}, \widetilde{v}) \sim(u, v)\right\} .
\end{aligned}
$$

Proof of Theorem 8.7. The finiteness of the relaxed energy $\widetilde{E_{\gamma}}[u, v]$ is a consequence of the arguments in Section 6. We prove separately the lower bound and the upper bound in equality (8.14).

Step 1. Lower bound. Let $\left\{\left(u_{k}, v_{k}\right)\right\}$ be a sequence of couples of smooth functions in $C^{\infty}\left(B^{n}, \mathbb{S}^{2}\right)$ such that $\left[u_{k}, v_{k}\right] \rightarrow[u, v]$ in $M_{2} W^{1,2}$, with $\sup _{k} E_{\gamma}\left[G_{u_{k}}, G_{v_{k}}\right]<\infty$. Arguing as in Proposition 8.6, we can assume that $E_{\gamma}\left[G_{u_{k}}, G_{v_{k}}\right]=\mathcal{E}_{\gamma}\left(S_{k}, T_{k}\right)$ for some $\left(S_{k}, T_{k}\right) \in\left[G_{u_{k}}, G_{v_{k}}\right]$ such that $S_{k} \rightarrow S$ and $T_{k} \rightarrow T$ in $\mathcal{D}_{n}\left(B^{n} \times \mathbb{S}^{2}\right)$ to some $S, T \in \operatorname{cart}^{2,1}\left(B^{n} \times \mathbb{S}^{2}\right)$. The lower semicontinuity implies that

$$
E_{\gamma}[S, T] \leq \mathcal{E}_{\gamma}(S, T) \leq \liminf _{k \rightarrow \infty} \mathcal{E}_{\gamma}\left(S_{k}, T_{k}\right)=\liminf _{k \rightarrow \infty} E_{\gamma}\left[G_{u_{k}}, G_{v_{k}}\right]
$$

Moreover, the weak convergence $\left[S_{k}, T_{k}\right] \rightarrow[S, T]$ in $M_{2} \operatorname{cart}^{2,1}\left(B^{n} \times \mathbb{S}^{2}\right)$ implies the weak convergence $\left[u_{S_{k}}, u_{T_{k}}\right] \rightarrow\left[u_{S}, u_{T}\right]$ in $M_{2} W^{1,2}\left(B^{n}, \mathbb{S}^{2}\right)$. But since $\left[u_{S_{k}}, u_{T_{k}}\right]=\left[u_{k}, v_{k}\right]$ we deduce that $\left(u_{S}, u_{T}\right) \sim(u, v)$. Together with (8.15) this proves the inequality " $\geq$ " in (8.14).

Step 2. Upper bound. Consider $\widetilde{u}, \widetilde{v} \in W^{1,2}\left(B^{n}, \mathbb{S}^{2}\right)$ such that $(\widetilde{u}, \widetilde{v}) \sim(u, v)$ and let $S \in \mathcal{T}_{\widetilde{u}}$ and $T \in \mathcal{T}_{\widetilde{v}}$. By Proposition 8.5, we have $E_{\gamma}[S, T]=\mathcal{E}_{\gamma}(\widetilde{S}, \widetilde{T})$ for some couple of currents $\widetilde{S}, \widetilde{T} \in \operatorname{cart}^{2,1}\left(B^{n} \times \mathbb{S}^{2}\right)$ such that $(\widetilde{S}, \widetilde{T}) \simeq(S, T)$. By Corollary 6.3, we take two sequences $\left\{u_{k}\right\},\left\{v_{k}\right\} \subset C^{\infty}\left(B^{n}, \mathbb{S}^{2}\right)$ such that $\left[G_{u_{k}}, G_{v_{k}}\right] \rightarrow[\widetilde{S}, \widetilde{T}]$ weakly in $M_{2}$ cart $^{2,1}$ and $\mathcal{E}_{\gamma}\left(G_{u_{k}}, G_{v_{k}}\right) \rightarrow \mathcal{E}_{\gamma}(\widetilde{S}, \widetilde{T})$. Arguing as in Proposition 8.6, we write again $E_{\gamma}\left[G_{u_{k}}, G_{v_{k}}\right]=$ $\mathcal{E}_{\gamma}\left(S_{k}, T_{k}\right)$ for some $\left(S_{k}, T_{k}\right) \in\left[G_{u_{k}}, G_{v_{k}}\right]$ such that $\left[S_{k}, T_{k}\right] \rightarrow[\bar{S}, \bar{T}]$ in $M_{2} \operatorname{cart}^{2,1}\left(B^{n} \times \mathbb{S}^{2}\right)$ with

$$
E_{\gamma}[\bar{S}, \bar{T}] \leq \mathcal{E}_{\gamma}(\bar{S}, \bar{T}) \leq \liminf _{k \rightarrow \infty} E_{\gamma}\left[G_{u_{k}}, G_{v_{k}}\right] \leq \lim _{k \rightarrow \infty} \mathcal{E}_{\gamma}\left(G_{u_{k}}, G_{v_{k}}\right)=\mathcal{E}_{\gamma}(\widetilde{S}, \widetilde{T})=E_{\gamma}[S, T]
$$


Since $\left[G_{u_{k}}, G_{v_{k}}\right]=\left[S_{k}, T_{k}\right]$, we have $(S, T) \simeq(\widetilde{S}, \widetilde{T}) \simeq(\bar{S}, \bar{T})$. This implies that (8.16) is a chain of equalities, and in particular

$$
\liminf _{k \rightarrow \infty} E_{\gamma}\left[G_{u_{k}}, G_{v_{k}}\right]=E_{\gamma}[S, T]
$$

Moreover, by the choice of $S$ and $T$, we also have

$$
\left(u_{\bar{S}}, u_{\bar{T}}\right) \sim\left(u_{S}, u_{T}\right)=(\widetilde{u}, \widetilde{v}) \sim(u, v),
$$

that also gives

$$
\left[u_{k}, v_{k}\right]=\left[u_{S_{k}}, u_{T_{k}}\right] \rightarrow\left[u_{\bar{S}}, u_{\bar{T}}\right]=[u, v]
$$

weakly in $M_{2} W^{1,2}$. This, together with (8.17), yields the inequality " $\leq$ " in (8.14).

\section{Couples of Functions Versus multifunctions}

This final section is devoted to a simple example showing the difference between the two viewpoints, couples of functions versus multifunctions: this implies in particular that the value of $\mathcal{E}_{\gamma}(u, v)$ depends on the choice of the representative in the equivalence class $[u, v]$. This motivates the definition of $\mathcal{E}_{\gamma}[u, v]$ given in (8.4).

The starting point is to remark that the union of the graphs of the functions (from $\mathbb{R}$ to $\mathbb{R}$ )

$$
u_{0}(x)=x^{+}, \quad v_{0}(x)=x^{-}
$$

may be reobtained with the two functions $\widetilde{u}_{0}(x)=|x|$ and $\widetilde{v}_{0}(x) \equiv 0$, and that the argument of the interaction integral in (1.4), near the point $x=0$, is always 1 when applied to the couple $\left(\widetilde{u}_{0}, \widetilde{v}_{0}\right)$, and is sometimes zero and sometimes 4 (due to the square) when applied to $\left(u_{0}, v_{0}\right)$ : thus depending on the sign of $\gamma$ either choice as a representative in the equivalence class may be more favorable than the other.

This does not instantly apply to our setting of functions from a ball to a sphere, but we show how to reproduce the same behavior. Define the Sobolev functions in $W^{1,2}\left(B^{n}, \mathbb{S}^{2}\right)$

$$
u(x):=\left\{\begin{array}{ll}
\left(\sin x_{1}, \cos x_{1}, 0\right) & \text { if } x_{1} \geq 0 \\
(0,1,0) & \text { if } x_{1}<0,
\end{array} \quad v(x):= \begin{cases}(0,1,0) & \text { if } x_{1} \geq 0 \\
\left(-\sin x_{1}, \cos x_{1}, 0\right) & \text { if } x_{1}<0\end{cases}\right.
$$

where $x=\left(x_{1}, x^{\prime}\right) \in B^{n}, x^{\prime}=\left(x_{2}, \ldots, x_{n}\right)$, and

$$
\widetilde{u}(x):=\left(\sin \left|x_{1}\right|, \cos x_{1}, 0\right), \quad \widetilde{v}(x):=(0,1,0),
$$

so that $(u, v) \sim(\widetilde{u}, \widetilde{v})$, see Definition 8.1, and remark that $|D \widetilde{u}(x)-D \widetilde{v}(y)|$ is identically equal to 1 , whereas $|D u(x)-D v(y)| \leq 2$ everywhere. Since

$$
\frac{1}{2} \int_{B^{n}}|D u(x)|^{2} \mathrm{~d} x+\frac{1}{2} \int_{B^{n}}|D v(x)|^{2} \mathrm{~d} x=\frac{1}{2} \int_{B^{n}}|D \widetilde{u}(x)|^{2} \mathrm{~d} x+\frac{1}{2} \int_{B^{n}}|D \widetilde{v}(x)|^{2} \mathrm{~d} x,
$$

we need only compare the values at $(u, v)$ and $(\widetilde{u}, \widetilde{v})$ of the last integral in (1.4), namely we consider

$$
\begin{aligned}
\Delta_{r}:= & \int_{B^{n}} f_{\widetilde{B}_{r}(x)} \frac{1}{2}\left(|D u(x)-D v(y)|^{2}+|D v(x)-D u(y)|^{2}\right) \mathrm{d} y \mathrm{~d} x \\
& -\int_{B^{n}} f_{\widetilde{B}_{r}(x)} \frac{1}{2}\left(|D \widetilde{u}(x)-D \widetilde{v}(y)|^{2}+|D \widetilde{v}(x)-D \widetilde{u}(y)|^{2}\right) \mathrm{d} y \mathrm{~d} x \\
= & \int_{B^{n}} f_{\widetilde{B}_{r}(x)}\left[\frac{1}{2}\left(|D u(x)-D v(y)|^{2}+|D v(x)-D u(y)|^{2}\right)-1\right] \mathrm{d} y \mathrm{~d} x \\
= & \int_{B^{n}} f_{\widetilde{B}_{r}(x)}\left(|D u(x)-D v(y)|^{2}-1\right) \mathrm{d} y \mathrm{~d} x \\
= & \int_{B^{n}} f_{\widetilde{B}_{r}(x)}\left(\left|D_{1} u(x)-D_{1} v(y)\right|^{2}-1\right) \mathrm{d} y \mathrm{~d} x,
\end{aligned}
$$


where in the second-last equality we exploited the peculiar symmetry of the couples $(u, v)$ and $(v, u)$ with respect to the transformation $\left(x_{1}, x^{\prime}\right) \mapsto\left(-x_{1}, x^{\prime}\right)$, and in the last one the fact that all functions only depend on $x_{1}$, and we have denoted by $D_{1}$ the derivative with respect to $x_{1}$. We will find a simple estimate as $r \rightarrow 0$, thus from now on we suppose $r$ small enough for all our geometrical considerations to hold true. For $\left|x_{1}\right|>r$ the integrand function vanishes on $B_{r}(x)$ and we have

$$
\Delta_{r}=\int_{A_{r}} f_{\widetilde{B}_{r}(x)}\left(\left|D_{1} u(x)-D_{1} v(y)\right|^{2}-1\right) \mathrm{d} y \mathrm{~d} x
$$

where we set $A_{r}:=\left\{x \in B^{n}:-r<x_{1}<r\right\}$. Now define

$$
C_{r}:=\left\{x \in A_{r}:\left\|\left(x_{2}, \ldots, x_{n}\right)\right\|<1-2 r\right\},
$$

a cylinder such that $\widetilde{B}_{r}(x)$ is just $B_{r}(x)$ whenever $x \in C_{r}$. Since the volume of $A_{r} \backslash C_{r}$ is of order $r^{2}$ and the integrand function is bounded (say, by 5 ) we may write

$$
\Delta_{r}=O\left(r^{2}\right)+\int_{C_{r}} f_{B_{r}(x)}\left(\left|D_{1} u(x)-D_{1} v(y)\right|^{2}-1\right) \mathrm{d} y \mathrm{~d} x
$$

Now set

$$
D_{1} u_{0}(x):=\left\{\begin{array}{l}
(1,0,0) \text { if } x_{1} \geq 0 \\
(0,0,0) \text { if } x_{1}<0,
\end{array} \quad D_{1} v_{0}(y):=\left\{\begin{array}{l}
(0,0,0) \text { if } y_{1} \geq 0 \\
(-1,0,0) \text { if } y_{1}<0
\end{array}\right.\right.
$$

and remark that if $x \in C_{r}$ we have

$$
D_{1} u(x)=D_{1} u_{0}(x)+O(r) \quad \text { and } \quad D_{1} v(y)=D_{1} v_{0}(y)+O(r) \quad \forall y \in B_{r}(x),
$$

thus

$$
\Delta_{r}=O\left(r^{2}\right)+\int_{C_{r}} f_{B_{r}(x)}\left(\left|D_{1} u_{0}(x)-D_{1} v_{0}(y)\right|^{2}-1\right) \mathrm{d} y \mathrm{~d} x=: O\left(r^{2}\right)+\int_{C_{r}} f_{B_{r}(x)} \phi(x, y) \mathrm{d} y \mathrm{~d} x
$$

as the volume of $C_{r}$ is of the order of $r$. We write down the values taken by this last integrand:

$$
\phi(x, y)= \begin{cases}0 & \text { if } x_{1}>0, y_{1}>0 \quad \text { or } \quad x_{1}<0, y_{1}<0 \\ 3 & \text { if } x_{1}>0, y_{1}<0 \\ -1 & \text { if } x_{1}<0, y_{1}>0\end{cases}
$$

Thus (computing the integral of the function $x_{1}$ on the half sphere $x_{1}>0$ )

$$
\Delta_{r}=O\left(r^{2}\right)+\frac{2}{\omega_{n} r^{n}} \int_{\left\{x \in C_{r} \mid x_{1}>0\right\}} \int_{\left\{y \in B_{r}(x) \mid y_{1}<0\right\}} 1 \mathrm{~d} x \mathrm{~d} y=O\left(r^{2}\right)+\frac{2 \omega_{n-1}^{2} r}{(n+1) \omega_{n}} .
$$

This proves in particular that $\Delta_{r}>0$ for all $r$ suitably small: thus, since

$$
\mathcal{E}_{\gamma}(u, v)-\mathcal{E}_{\gamma}(\widetilde{u}, \widetilde{v})=\gamma \Delta_{r}
$$

we deduce for $r$ small

$$
\begin{aligned}
& \gamma>0 \quad \Rightarrow \quad \mathcal{E}_{\gamma}(\widetilde{u}, \widetilde{v})<\mathcal{E}_{\gamma}(u, v) \\
& \gamma<0 \quad \Rightarrow \quad \mathcal{E}_{\gamma}(u, v)<\mathcal{E}_{\gamma}(\widetilde{u}, \widetilde{v}) .
\end{aligned}
$$


Since moreover $\widetilde{u}(x)=\widetilde{v}(x)$ if and only if $x_{1}=0$, by Definition 8.2 it is possible to check that the equivalence class $[u, v] \in M_{2} W^{1,2}\left(B^{n}, \mathbb{S}^{2}\right)$ has only two couples of representatives. We have thus obtained that for $r$ small the energy (8.4) of such an equivalence class satisfies

$$
E_{\gamma}[u, v]=\left\{\begin{array}{l}
\mathcal{E}_{\gamma}(\widetilde{u}, \widetilde{v}) \text { if } \gamma \geq 0 \\
\mathcal{E}_{\gamma}(u, v) \text { if } \gamma<0 .
\end{array}\right.
$$

Finally, since all the currents $G_{w}$ carried by the graphs of the functions $w=u, v, \widetilde{u}, \widetilde{v}$ in our example satisfy the null-boundary condition (3.3), hence belong to the class $\operatorname{cart}^{2,1}\left(B^{n} \times \mathbb{S}^{2}\right)$, by Theorem 8.7 we conclude that for $\gamma>\max \left\{\gamma_{s}(r), \gamma_{c}(r)\right\}$ the relaxed energy (8.12) always satisfies

$$
\widetilde{E_{\gamma}}[u, v]=E_{\gamma}[u, v]=\left\{\begin{array}{l}
\mathcal{E}_{\gamma}(\widetilde{u}, \widetilde{v}) \text { if } \gamma \geq 0 \\
\mathcal{E}_{\gamma}(u, v) \text { if } \gamma<0 .
\end{array}\right.
$$

\section{REFERENCES}

[1] F.J. Almgren, W. Browder and E.H. Lieb, Co-area, liquid crystals, and minimal surfaces, in Partial Differential Equations, Lecture Notes in Math. 1306. Springer (1988) 1-22.

[2] F. Bethuel, The approximation problem for Sobolev maps between manifolds. Acta Math. 167 (1992) $153-206$.

[3] H. Brezis, J.M. Coron and E.H. Lieb, Harmonic maps with defects. Comm. Math. Phys. 107 (1986) 649-705.

[4] H. Federer, Geometric measure theory, Grundlehren Math. Wissen. 153. Springer, New York (1969).

[5] H. Federer and W. Fleming, Normal and integral currents. Annals of Math. 72 (1960) 458-520.

[6] M. Giaquinta and G. Modica, On sequences of maps with equibounded energies. Calc. Var. 12 (2001) $213-222$.

[7] M. Giaquinta, G. Modica and J. Souček, Cartesian currents in the calculus of variations, I, II, Ergebnisse Math. Grenzgebiete (III Ser.) 37, 38. Springer, Berlin (1998).

[8] M. Giaquinta and D. Mucci, Density results relative to the Dirichlet energy of mappings into a manifold. Comm. Pure Appl. Math. 59 (2006) 1791-1810.

[9] M. Giaquinta and D. Mucci, Maps into manifolds and currents: area and $W^{1,2}-, W^{1 / 2}-, B V$-energies, Edizioni della Normale. C.R.M. Series, Sc. Norm. Sup. Pisa (2006).

[10] J. Sacks and K. Uhlenbeck, The existence of minimal immersions of 2-spheres. Annals of Math. 113 (1981) 1-24.

[11] R. Schoen and K. Uhlenbeck, Boundary regularity and the Dirichlet problem for harmonic maps. J. Diff. Geom. 18 (1983) 253-268.

[12] L. Simon, Lectures on geometric measure theory, Proc. of the Centre for Math. Analysis 3. Australian National University, Canberra (1983).

[13] U. Tarp-Ficenc, On the minimizers of the relaxed energy functionals of mappings from higher dimensional balls into $\mathbb{S}^{2}$. Calc. Var. Partial Differential Equations 23 (2005) 451-467.

[14] E.G. Virga, Variational theories for liquid crystals, Applied Mathematics and Mathematical Computation 8. Chapman \& Hall, London (1994). 